\title{
OPTIMAL STOPPING AND FREE BOUNDARY PROBLEMS
}

PIERRE VAN MOERBEKE ${ }^{1}$

Consider a random situation or a game where at each moment a gambler is to make one of two decisions: quitting or pursuing the game purely on the basis of present information; his only opponent - so to speak - is randomness. Both decisions are not equally favorable, but their efficiency depends on the unknown outcome of the game. A reward function measures this efficiency: the higher its value, the better the situation from the gambler's viewpoint. Hence he has to decide whether the future gain will outweigh the loss due to stopping or further unfavorable moves. We aim at studying the decision rules or strategies yielding the best possible average gain. The purpose of this lecture is to show the relationship between the optimal stopping problem and a free boundary value problem for the heat equation. The study of this connection has led to a deeper grasp of various natural questions about the problem and to a more qualitative description of its solutions, opposed to a discrete approach which of course would lend itself much better to numerical results.

This is not a streamlined, but rather a cursory and leisurely account on the subject; proofs and further details may be found in the author's papers $[46,47]$. Several excellent books and articles written on this theme have encouraged me to omit other interesting aspects. These are the recent books by $\mathrm{H}$. Robbins and D. Siegmund [37] and A. N. Širjaev [42] and the articles by L. Breiman [8] and H. Chernoff [11, $12,13,14]$.

1. Introduction. During the last decade, various authors have addressed themselves to such problems in the context of statistical decision theory, operations research and game theory. We mention here a few of these problems:

1. There is the well-known "secretary problem", which made its debut with $\mathrm{D}$. V. Lindley [30] and received a rigorous treatment from Y. S. Chow, S. Moriguti, H. Robbins and S. M. Samuels [15] .

Let $n$ secretaries apply for a job. They are to be graded according to quality from the best (1) to the worst $(n)$. There is only a single position available and the interviewer can compare the present appli-

Received by the Editors June 22, 1973.

1Supported in part by NSF grant GP-36418X, while a member of the Institute for Advanced Study, Princeton, New Jersey 08540.

Copyright 1974 Rocky Mountain Mathematics Consortium 539 
cant only to the ones he has seen to date. After each interview, two alternatives are open to him: he can accept the candidate or he can reject him or her and proceed to the next interview. It is understood that no one rejected can be recalled. What will be the optimal strategy so as to maximize the expected ability of the secretary so chosen? Moreover, what will be the expected rank of the candidate selected in this way?

In this problem, the random quantities $y_{i}$ to be considered are $y_{i}=1+$ the number of previous candidates better than the one seen at the $i$ th interview. The variables $y_{i}$ are independent, because if you have picked already $i-1$ numbers $x_{1} \cdots x_{i-1}$ out of the set $\{1, \cdots, n\}$ and ifyou have placed them in order $x_{k_{1}}<x_{k_{2}}<\cdots<x_{k_{i-1}}$, then the probability that the $i$ th number chosen will sit between, say, $x_{k_{j}}$ and $x_{k_{j+1}}$ is $1 / i$ independently of the order in which you have taken the $i-1$ first numbers. Given $y_{i}=j$, a simple combinatorial argument gives the average rank of the candidate selected at the $i$ th interview, namely,

$$
\frac{n+1}{i+1} y_{i}
$$

Hence the optimal strategy $\tau$ is the one which minimizes

$$
E \frac{n+1}{\tau+1} y_{\tau}
$$

it is described by a sequence of numbers $\left(s_{1}, \cdots, s_{n}\right)$, and the rule is: hire as soon as $y_{i} \leqq s_{i}$.

Where do these numbers come from? They result from a backward induction procedure. Here is the idea of this induction: hiring someone is required, so at the last interview the average rank is the mean quality of the $n$ possible secretaries. At the previous interview, the interviewer is facing two alternatives: hiring at once or waiting for the next interview. Hence for each value of $y_{n-1}$, he compares the quality of the candidate selected with the average quality in case he would go on. The first value of $y_{n-1}$ for which proceeding to the next interview is disadvantageous determines $s_{n-1}$. The determination of $s_{n-2}$ is similar, etc. $\cdots$.

For instance, if $n=4$, the optimal strategy is to always reject the first candidate, to appoint the second, if he or she is better than the first and to appoint the third one, if at most one previously interviewed is better; finally, in the last interview, you always appoint, since you have to appoint someone. The expected rank of the candidate selected using this strategy is 1.875 . 
Evidently, the selection strategy will depend on the total number $n$ of candidates. But the expected rank of the candidate selected in this way has the surprising virtue never to be worse than 4 . In fact, when the number $n$ of candidates tends to $\infty$, the optimal expected rank increases to

$$
\prod_{j=1}^{\infty}\left(\frac{j+2}{j}\right)^{1 /(j+1)}=3.8695
$$

2. An urn contains white and red balls. We must predict which is the dominant color by drawing balls one at the time with replacement. For each ball drawn we must pay one dollar and we are to receive $A$ dollars for a correct guess and nothing for an incorrect guess. The aim is to maximize our payoff. What is the optimal strategy?

To see how this problem may be solved, first consider a random walk on the lattice of Figure 1 according to the rule: jump one step upwards or one step to the right according as the color of the ball drawn is red or white. A bayesian hypothesis is needed to determine

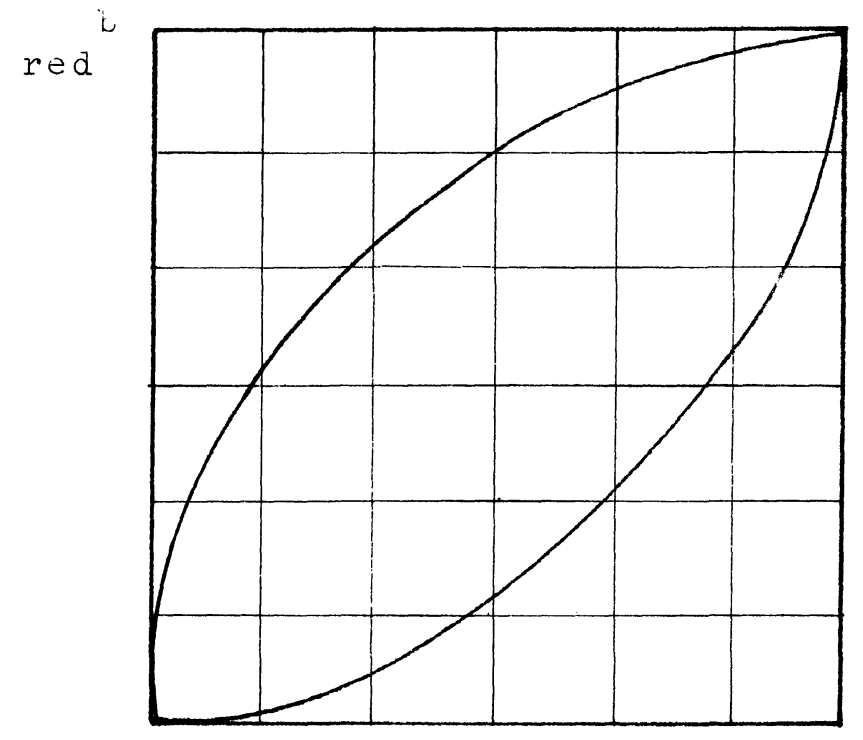

Fig. ]

the a posteriori probability of drawing a ball of a certain color given the sample already obtained. This probability is the transition prob- 
ability of the random walk. The optimal policy can be described as follows: sample as long as the walk remains inside a certain pearshaped region; stop when it hits the boundary. Then decide white or red according to the majority at that time. This example is taken from S. Moriguti and $\mathrm{H}$. Robbins [34]. They also consider the behavior of the pear-shaped curve when $A$ and the total number $(a$, of white; $b$, of red) of balls tend to infinity in such a way that:

$$
\frac{a+b}{A^{2 / 3}} \text { and } \frac{a-b}{A^{1 / 3}}
$$

remain finite. It is natural to think of the limits of these ratios as time $(t)$ and position $(x)$. Taking such a limit, the random walk tends to a diffusion and the pear-shaped curve is replaced by a continuous curve $x= \pm s(t)$ as pictured in Figure 2 .

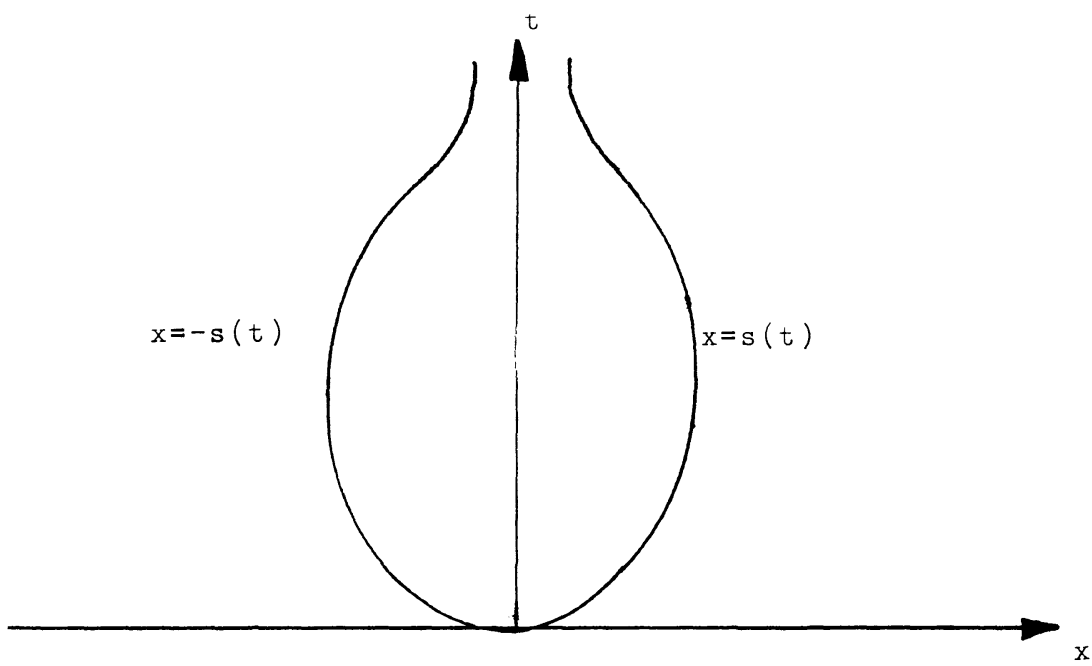

Fig. 2

Moriguti and Robbins prove that for large $t$

$$
s(t) \simeq \frac{1}{4 t}-\frac{1}{48 t^{4}}+\frac{7}{960 t^{7}}, t \nearrow \infty .
$$

J. A. Bather [2] has considered the problem of deciding the sign of a normal mean with known variance. As expected an appropriate limiting procedure leads to the same continuous problem.

In a series of papers, Chernoff $[11,12,13]$ and Breakwell and Chernoff [7] dealt with the problem of guessing from continuous observations the sign of the (constant) drift coefficient $\mu$ of a brownian 
motion $b t+\mu t, 1$ being the cost per observation and 1 the cost of an incorrect decision. Introducing the appropriate bayesian hypothesis, they prove that the estimated drift evolves according to the diffusion of Moriguti and Robbins, or approximately so. The asymptotic expansion of the optimal sampling curve, calculated immediately from the continuous problem, gives the same result as (1) for large $t$. Chernoff [12] also performed an asymptotic expansion on the optimal sampling curve for small $t$ and obtained

$$
s(t) \sim \sqrt{t\left(3 \log \frac{1}{t}-\log \frac{8 \pi}{3}\right)}, t \searrow 0 .
$$

We refer the interested reader to an excellent review paper by $\mathrm{H}$. Chernoff [14].

3. In an unlimited fair coin-tossing game, a head contributes one dollar to your fortune and a tail makes you lose one dollar. However, inflation depreciates your initial stake of one dollar to a value $c_{n}<1$ dollar at time $n$ and $c_{1}>c_{2}>\cdots>c_{n}$ decreases to 0 as $n>\infty$. After the $n$th toss you have two alternatives: either take your chances and continue the game one more time, or quit the game and collect your gain to date, namely, $c_{n}\left(x_{1}+\cdots+x_{n}\right)$, in which $x_{k}= \pm 1$ is the outcome of the $k$ th toss.

When will you decide to quit the game in order to achieve the highest expected gain? If you wait too long and the depreciation rate of your currency is very high, you may lose everything. On the contrary, if you toss the coin only a few times, the game may not be in your favor.

In the case of $c_{n}=1 / n, \mathrm{Y}$. S. Chow and H. E. Robbins [16] have proved the existence of an optimal stopping rule. A. Dvoretzky [19], H. Teicher and J. Wolfowitz [45] proved the existence of an optimal stopping rule, when the $X_{i}$ are independent identically distributed random variables with zero mean and variance 1 : there is a series of specific constants $\beta_{1}<\beta_{2}<\cdots<\beta_{n}<\cdots$, such that the best policy is to quit the game as soon as $S_{n}=\sum_{i=1}^{n} X_{i} \geqq \beta_{n}$. Dvoretzky proves that

$$
0.32 \cdots \leqq \frac{\beta_{n}}{\sqrt{n}} \leqq 4.06 \cdots
$$

for $n$ large enough, and he conjectures that

$$
\lim _{n \uparrow \infty} \frac{\beta_{n}}{\sqrt{n}}
$$


exists. This conjecture was proved independently by H. M. Taylor [44], L. H. Walker [48] and L. A. Shepp [41], who found 0.839 as limiting value. They point out that considered for large $n$ 's this problem and the brownian analogue must be equivalent. The brownian analogue is to maximize

$$
E \frac{x_{\tau}}{t+\tau}
$$

over all non-anticipating random times $\tau$, where $x_{\tau}$ denotes brownian motion starting at $x_{0}=0$ evaluated at time $\tau$. Shepp proves that the best policy is to stop as soon as

$$
x_{s} \geqq \alpha \sqrt{t+s},
$$

where $\alpha$ is the same as the limiting value of $\beta_{n} / \sqrt{n}$ in the discrete problem. In the case where $c_{n}=e^{-n}$, the optimal policy is to stop as soon as $S_{n} \geqq C$, for some appropriate constant $C$. The brownian analogue here is to maximize

$$
E x_{\tau} e^{-\beta(t+\tau)},
$$

over all random times $\tau$. The best policy is to stop as soon as $x_{s} \geqq$ $1 / \sqrt{2}$.

Shepp's or Walker's approach to the continuous version of the former problems is by discretization. It may be solved more advantageously by the so-called "smooth-fit" property, to be explained below. The idea was already in the air since D. V. Lindley [30]. There is indeed a vast class of problems leading to parabolic optimal boundaries which are easily treated by this method, as we shall discuss later; much of our attention is therefore devoted to this phenomenon.

4. H. P. McKean [33] and P. A. Samuelson [39] consider the problem of rational pricing of stock warrants. A warrant is a contract conferring the right to purchase a common stock at a fixed unit price at any time previous to some date of expiration. Both the expiration date (which may be infinite for "perpetual warrants") and the unit price are stipulated in the contract. Samuelson's model for the stock price fluctuations is a logarithmic brownian motion, $e^{\sigma x(t)+\delta t}$, where $x(t)$ denotes the ordinary brownian motion and $\sigma$ and $\delta$ are constants. In this problem too, the optimal buying policy can be worked out. We shall come back to it in $\S 3$.

2. A Brief Excursion Through the Theory. What is the essence of all these problems? They involve a time $t$ and a position $x$ measuring the "state of affairs" such as the quantity $y_{i}$ in the "secretary problem", 
or the number of red and white balls in the sample, or the fortune in the coin-tossing game or the current price of the stock in the warrant problem; in each of these problems, $x$ fluctuates in a Markovian fashion. For the sake of simplicity we deal only with the case in which $x$ moves according to a brownian motion, or approximately so. This is not so special a problem, as it may seem; it is in fact typical and most of the problems studied to date either exhibit a brownian behavior, genuine or transformed, or can be so approximated by a suitable scaling, provided only that the number of trials is very large.

The game is now specified by fixing the "reward function" $g=$ $g(x, t)$, with the following meaning: if the gambler decides to quit at time $t$ when his state of affairs is $x$, his reward will be $g(x, t)$. Contrariwise, if he decides to play for a possibly random period of time $\tau$, his average reward would be

$$
E g\left(x+x_{\tau}, t+\tau\right)
$$

where $x(t)$ is the brownian motion starting at $x(0)=0$. Notice that $\left(x+x_{s}, t+s\right)$ is the customary space-time brownian motion starting at $(x, t)$; it is the graph of the usual brownian motion, as in Figure 3.

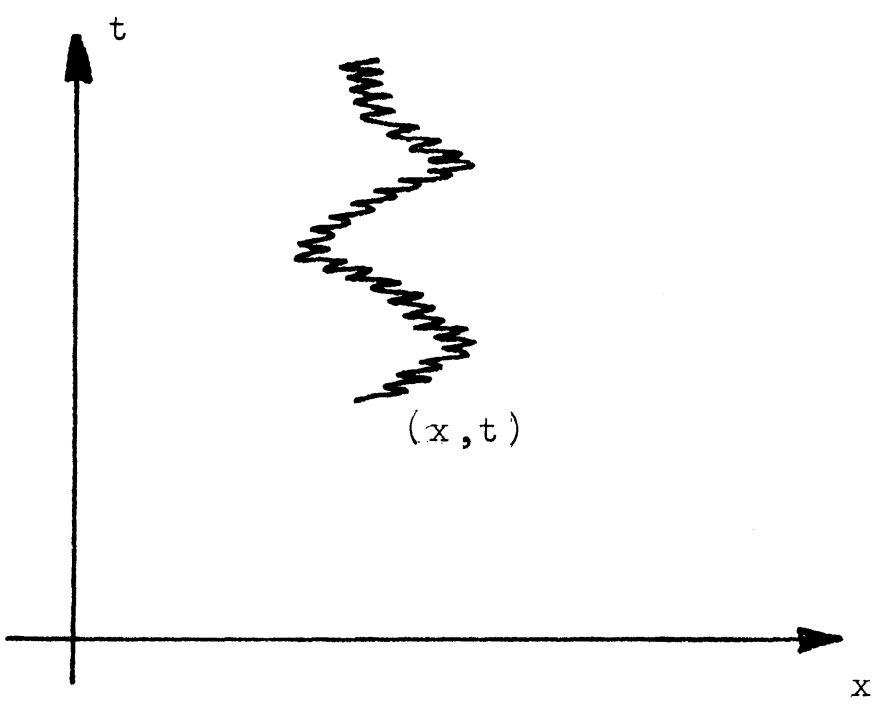

Fig. 3

Of course, it is not permitted to the gambler to foresee the future. This is built in by allowing only "stopping times" $\tau$, by which you will understand that the event $\tau \leqq t$ depends upon the brownian motion up to time $t$ only and not upon what it does afterwards, e.g., the first 
hitting time of 1 is a stopping time as opposed to the least leaving from 0 before time $t=1$, which depends on the future.

Henceforth, we will refer to two kinds of games, one where the playing time is bounded above by some $T<\infty$ (games with a finite horizon) and games where one can play for ever (games with an infinite horizon).

Unless specified otherwise, we assume $g$ and all its partials to be continuous for $t<T$ and to have limits, as $t \nearrow T$; a discontinuity at $t=T$ is permitted, if $T<\infty$, but $h(x)=g(x, T)-(x, T-)$ is to be infinitely differentiable, except perhaps for a few isolated jumps ( $g(x, T-)$ means the left limit of $g(x, t)$ at $t=T) ; h$ will be called the final gain. You may assume $h(x) \geqq 0$; the motivation is that if $h(x)<0$ in an interval, it is more favorable to stop the game a little before hitting the final horizon $t=T$.

The optimal reward $\hat{g}(x, t)$ starting at time $t$ and with a state of affairs $x$ is now obtained by maximizing

$$
E g\left(x+x_{\tau}, t+\tau\right),
$$

over all stopping times $\tau$, if the horizon is infinite and over all stopping times $\tau \leqq T-t$ in a game with finite horizon $T$. The optimal strategy is the stopping time $\tau$ achieving this maximum.

2.1 Alternative Characterizations of $\hat{g}$.

(a) $\hat{g}$ is the smallest excessive function exceeding $g$. A function $f$, bounded below, is called excessive in an open domain of $R^{2}$ if

(i) $E f\left(x+x_{\tau}, t+\tau\right) \leqq f(x, t)$ for every stopping time $\tau$ not exceeding the first exit time $\tau_{D}$ from $D$.

(ii) $E f\left(x+x_{\tau_{n}}, t+\tau_{n}\right) \nearrow f(x, t)$ for every sequence of stopping times $\tau_{n}<\tau_{D}$ such that $P\left(\tau_{n} \downarrow 0\right)=1$.

Notice that, if $f$ is moreover continuous, excessivity is actually a local property and, if $f$ is sufficiently differentiable, excessivity in the domain $D$ is the same as

$$
\frac{\partial f}{\partial t}+\frac{1}{2} \frac{\partial^{2} f}{\partial x^{2}} \leqq 0
$$

for all $(x, t)$ in $D$.

(b) The backward induction for the discrete problem, as indicated in the "secretary problem" in $\S 1$, is adapted to the present case in the form

$$
\hat{g}(x, t)=\sup _{n} g_{n}(x, t)
$$

where $g_{0}=g$ and 


$$
g_{n}(x, t)=\sup _{s \geqq 0} E g_{n-1}\left(x+x_{s}, t+s\right) .
$$

The proof that $\hat{g}=\sup g_{n}$ is in one line: you have $\hat{g} \geqq \sup g_{n} \geqq g$ and then you check that sup $g_{n}$ is excessive and use (a).

Both versions of the optimal stopping problem were already investigated by L. Snell [43], in the context of martingales.

2.2 The Appell Transformation. Except for the examples, this paper deals chiefly with finite horizon games, as the latter can be mapped into the former by the Appell transformation, all desired properties being carried over automatically. P. Appell [1] pointed out that if $u$ satisfies the heat equation, then

$$
v(x, t)=\frac{\frac{1}{t} u\left(\frac{x}{t}, \frac{1}{t}\right)}{\frac{1}{(2 \pi t)^{1 / 2}} \exp \left(-x^{2} / 2 t\right)}
$$

satisfies the backward heat equation.

This transformation is applied as follows: if $g$ is a reward function with infinite horizon, then

$$
g^{*}(x, t)=\frac{1}{\sqrt{2 \pi(T-t)}} \exp \left(-x^{2} / 2(T-t)\right) g\left(\frac{x}{T-t}, \frac{1}{T-t}\right), t \leqq T
$$

is a finite horizon game; $T$ is introduced in this expression as an artifact. Then the optimal reward $\hat{g}^{*}$ is related to $\hat{g}$, as follows:

$$
\hat{g}^{*}(x, t)=\frac{1}{(2 \pi(T-t))^{1 / 2}} \exp \left(-x^{2} / 2(T-t)\right) \hat{g}\left(\frac{x}{T-t}, \frac{1}{T-t}\right) \text {. }
$$

The proof of this identity is based on the connection between the Appell transformation and Doob's [18] $H$-processes or constrained processes.

2.3 The Tychonov Condition. For future convenience, we impose upon $g$ a growth condition, called the Tychonov condition; it is quite reminiscent of the Tychonov bounds for the uniqueness of solutions to the heat equation. We distinguish between the finite and infinite horizon case.

(a) $T<\infty$.

If the functions

$$
\begin{gathered}
g, \frac{\partial g}{\partial t}, \frac{\partial g}{\partial x}, \frac{\partial^{2} g}{\partial x^{2}}, \frac{\partial^{2} g}{\partial x \partial t}, \frac{\partial^{3} g}{\partial x^{3}} \\
\text { and } h, \frac{\partial h}{\partial x}, \frac{\partial^{2} h}{\partial x^{2}}, \text { and } \frac{\partial^{3} h}{\partial x^{3}}
\end{gathered}
$$


are bounded by $e^{o\left(x^{2}\right)}$ when $|x|$ tends to $\infty$, uniformly in any strip of finite depth $[t, T]$, then $g$ is said to satisfy the Tychonov condition.

(b) $T=\infty$.

$g$ satisfies the Tychonov condition if

$$
g^{*} \equiv \frac{1}{(2 \pi(-t))^{1 / 2}} \exp \left(-x^{2} / 2(-t)\right) g\left(\frac{x}{-t}, \frac{1}{-t}\right), t<0,
$$

satisfies the condition under (a) with $T=0$.

The Tychonov condition implies that $\hat{g}$ is finite and continuous.

2.4 The Payoff-Rate. Throughout this paper, the payoff-rate per unit time

$$
H \equiv \frac{\partial g}{\partial t}+\frac{1}{2} \frac{\partial^{2} g}{\partial x^{2}},
$$

plays an important role. The discrepancy between what you get if you would play for a period of time $\tau$ and what you get by not playing at all can be expressed only in terms of $H$ and the final gain $h$. Indeed, for $\tau \leqq T-t$

$$
\begin{aligned}
& E g\left(x+x_{\tau}, t+\tau\right)-g(x, t) \\
& \quad=E g\left(x+x_{\tau}, t+\tau-\right)-g(x, t) \\
& \quad+E\left(g\left(x+x_{\tau}, t+\tau\right)-g\left(x+x_{\tau}, t+\tau-\right) ; t+\tau=T\right)
\end{aligned}
$$

by Ito's lemma,

$$
\begin{gathered}
g\left(x+x_{\tau}, t+\tau\right)-g(x, t) \\
=\int_{0}^{\tau} \frac{\partial g}{\partial x}\left(x+x_{s}, t+s\right) d s+\int_{0}^{\tau} H\left(x+x_{s}, t+s\right) d s .
\end{gathered}
$$

But since $g$ satisfies the Tychonov condition, you have

$$
E \int_{0}^{T-t}\left|\frac{\partial g}{\partial x}\left(x+x_{s}, t+s\right)\right|^{2} d s<\infty,
$$

which implies that the expectation of the first term on the right-hand side of $(2)$ vanishes (cf. H. P. McKean [32], § 2.3.5). Hence,

$$
\begin{aligned}
E g\left(x+x_{\tau}, t+\tau\right)-g(x, t)= & E \int_{0}^{\tau} H\left(x+x_{s}, t+s\right) d s \\
& +E\left(h\left(x+x_{\tau}, t+\tau\right) ; t+\tau=T\right) .
\end{aligned}
$$

Using the Appell transformation, the same formula is shown to hold for $T=\infty$, except that then the term containing $h$ is absent. 
2.5 The Continuation and the Stopping Region. Now we distinguish two regions: a continuation region $C$ where $\hat{g}>g$, i.e., where it pays to play the game (which is open, since $\hat{g}$ is continuous) and a stopping region $S$ where $\hat{g}=g$, i.e., where quitting is best. The boundary separating these two regions is the optimal (stopping) boundary. Thanks to the Tychonov condition on $g$, the optimal strategy is to play as long as you remain in the continuation region and stop as soon as you hit the optimal boundary. If $\tau_{0}$ denotes this hitting time, this means that

$$
\hat{\mathrm{g}}(x, t)=E g\left(x+x_{\tau_{0}}, t+\tau_{0}\right) .
$$

From the previous section, it is obvious that one never stops at points where $H>0$, as proceeding a little while improves one's gain. Often stopping at points where $H=0$ is also unfavorable; for instance, when $\partial H / \partial x \neq 0$ at such points, or when $\partial H / \partial x=0$ and

$$
H^{2} \equiv\left(\frac{\partial}{\partial t}+\frac{1}{2} \frac{\partial^{2}}{\partial x^{2}}\right) H>0
$$

etc. $\cdots$.

2.6 A Free Boundary Problem for the Backwards Heat EquaTION. Evidently the ultimate aim is to find the optimal stopping boundary. So far we know that if $g$ satisfies the Tychonov condition, then stopping as soon as you enter the stopping region is optimal. But this merely describes the boundary in an implicit and somewhat circular way, in terms of $\hat{g}$, which we do not know yet. We now turn to analytical characterizations of $g$ in order to provide an effective way of computing the boundary.

The problem of finding $\hat{g}$ and the optimal strategy is to be converted into a free boundary problem for the heat equation. Why a free boundary problem? From (3), it follows that $\hat{g}$ is parabolic in the $C$-region. This means that

(i) $\hat{g}$ is excessive and

(ii) that $\hat{g}$ has the mean value property, i.e., $E \hat{g}\left(x+x_{\tau_{U}}, t+\tau_{U}\right)$ $=\hat{g}(x, t)$, where $\tau_{U}$ is the first exit time from any open set $U$ with compact closure.

Parabolic functions are shown to be $C^{\infty}$ and to satisfy the backwards heat equation; conversely, solutions of the backwards heat equation which are bounded below are all parabolic functions. Hence, you have

$$
\frac{\partial \hat{g}}{\partial t}+\frac{1}{2} \frac{\partial^{2} \hat{g}}{\partial x^{2}}=0 \text { in } C
$$




$$
\begin{gathered}
\hat{g}=g \text { at the boundary of } C, \\
\text { and } \hat{g}(x, T)=g(x, T) .
\end{gathered}
$$

Recall also from the previous section that $H \leqq 0$ in the S-region.

At this point we must assume that the continuation region $C$ has a continuously differentiable boundary $x=s(t)$, except for a few isolated points where $|s|$ may blow up. (The proof of this fact will actually be sketched in $\$ 4$ for rewards of the type $g=T-t, t<T$ and $=h(x)$, $t=T)$. Under this assumption, g's optimality implies that also the derivatives match, i.e.,

$$
\begin{aligned}
& \frac{\partial \hat{g}}{\partial x}(s(t), t)=\frac{\partial g}{\partial x}(s(t), t), \\
& \frac{\partial \hat{g}}{\partial x}(s(t), t)=\lim _{\substack{(y, u) \rightarrow(s(t), t) \\
(y, u) \in C}} \frac{\partial \hat{g}}{\partial x}(y, u),
\end{aligned}
$$

at points $(s(t), t)$, where $|\dot{s}|<\infty$. This should come as no surprise, because a moment's reflection shows that if this was not true you would always be able to improve your gain by playing a bit longer.

We are facing an initial and boundary value problem with two boundary conditions. This problem is overdetermined unless you agree to keep the boundary "free". So it seems plausible that (4), (5), (6) and (7) will determine the boundary $s(t)$ and the optimal reward g. The relations (6) and (7) will be called the smooth fit relations.

What is perhaps more important, since it gives us a recipe to compute examples, the converse of this statement is true (which is stated for $T<\infty)$.

THEOREM I. Let $C$ be an open set in $t \leqq T<\infty$ with a continuously differentiable boundary curve $x=s(t)$, except possibly for a finite number of isolated points where $\dot{s}$ blows up. Let a Tychonov-type function u satisfy

$$
\begin{aligned}
& \frac{\partial u}{\partial t}+\frac{1}{2} \frac{\partial^{2} u}{\partial x^{2}}=0 \text { in } C, \\
& u=g \text { at }(x, t)=(s(t), t), u(x, T)=g(x, T), \\
& \frac{\partial u}{\partial x}=\frac{\partial g}{\partial x} a t(x, t)=(s(t), t), \text { if }|\dot{s}|<\infty, \\
& u>g \text { in } C, u=g \text { elsewhere, and } H \leqq 0 \text { in the complement of } C .
\end{aligned}
$$

Then $u$ is actually $\hat{g}$ and $s(t)$ the optimal stopping boundary. 
2.7 The Integral Equation for the Optimal Boundary. The free boundary problem (4), (5), (6), and (7) can be converted into a Stefantype problem by considering instead of $\hat{g}$ the function $v=$ $\partial / \partial t(\hat{g}-g)$. It is easily checked that

$$
\begin{aligned}
& \frac{\partial v}{\partial t}+\frac{1}{2} \frac{\partial^{2} v}{\partial x^{2}}=-\frac{\partial H}{\partial t}(x, t) \text { in } C, \\
& v(s(t), t)=0,
\end{aligned}
$$$$
\frac{\partial v}{\partial x}(s(t), t)=2 H(s(t), t) \dot{s}(t),
$$

$$
\text { and } v(x, T)=-\frac{1}{2} \frac{\partial^{2} h}{\partial x^{2}}(x)-H(x, T-), \text { for }(x, T) \in \bar{C} \text {. }
$$

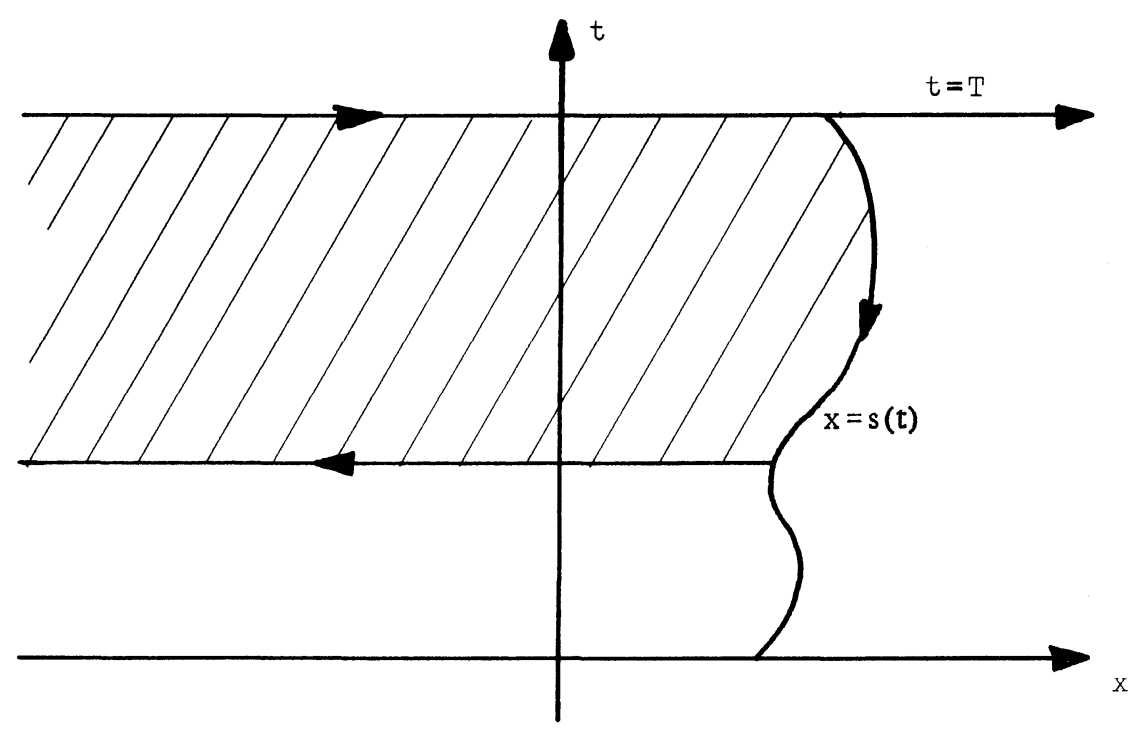

Fig. 4

For conversation's sake, take the continuation region to be bounded on one side (say, the right side) by a curve $x=s(t)$. The free boundary value problem (8) yields an integral equation for the boundary by applying Green's formula in the shaded part of the continuation region (as in Figure 4) to a form involving the Gauss kernel and the solution $v$, to obtain 


$$
\begin{aligned}
& H(s(t), t) \dot{s}(t)=-\int_{0}^{T-t} d \tau \\
& \quad \int_{-\infty}^{s(T-\tau)} \frac{\partial H}{\partial t}(\xi, T-\tau) \frac{s(t)-\xi}{(2 \pi(T-t-\tau))^{3 / 2}} \exp \left[-\frac{(s(t)-\xi)^{2}}{2(T-t-\tau)}\right] d \xi \\
& -\int_{0}^{T-t} \frac{s(t)-s(T-\tau)}{(2 \pi(T-t-\tau))^{3 / 2}} \exp \left[-\frac{(s(t)-s(T-\tau))^{2}}{2(T-t-\tau)}\right] \\
& \begin{array}{l}
\text { (9) } \\
H(s(T-\tau), T-\tau) \dot{s}(T-\tau) d \tau
\end{array}
\end{aligned}
$$

$$
\begin{aligned}
-\int_{-\infty}^{s(T)} \frac{s(t)-\xi}{(2 \pi(T-t))^{3 / 2}} \exp \left[-\frac{(s(t)-\xi)^{2}}{2(T-t)}\right] & \\
& \left(-\frac{1}{2} \frac{\partial^{2} h}{\partial \xi^{2}}(\xi)-H(\xi, T-)\right) d \xi .
\end{aligned}
$$

If the continuation region would be bounded on two sides by $x=$ $s_{1}(t)$ and $x=s_{2}(t)$, (9) would become a system of two integral equations in $s_{1}(t)$ and $s_{2}(t)$. Moreover, the right-hand side would acquire an additional term.

2.8 Relation to the Ice-Melting or -Freezing Problem. Consider the special reward $g=T-t$ for $t<T$ and $=h(x)$ for $t=T$. Let $w(x, \tau)=-v(x, T-\tau)$ and $\sigma(\tau)=s(T-\tau)$. Then the free boundary problem (8) simplifies to

$$
\begin{aligned}
& \frac{\partial w}{\partial \tau}=\frac{1}{2} \frac{\partial^{2} w}{\partial x^{2}} \text { in } C \\
& w(\boldsymbol{\sigma}(\tau), \tau)=0, \frac{\partial w}{\partial x}(\boldsymbol{\sigma}(\tau), \tau)=-2 \dot{\sigma}(\tau), \text { and } \\
& w(x, 0)=\frac{1}{2} \frac{\partial^{2} h}{\partial x^{2}}-1, \text { for }(x, 0) \in \bar{C}
\end{aligned}
$$

$w$ satisfies the heat equation, vanishes at the boundary; its flux through the boundary is proportional to the rate of change of the boundary and $(1 / 2)\left(\partial^{2} h / \partial x^{2}\right)-1$ stands for the initial temperature. If you will visualize the interval $x<\sigma(0)$ as filled with water at temperature $w(x, 0)$ and $x \geqq \sigma(0)$ with ice at zero temperature, then the boundary $x=\sigma(\tau)$ is the curve described by the interface between water and ice.

If the initial temperature $w(x, 0)=(1 / 2)\left(\partial^{2} h / \partial x^{2}\right)-1>0$, then the ice can only melt and it is obvious that the boundary $\sigma(\tau)$ must be increasing as in Figure 5. If $w(x, 0)$ is negative, then one must consider the water as being supercooled, i.e., below $0^{\circ} \mathrm{C}$. Then the water 


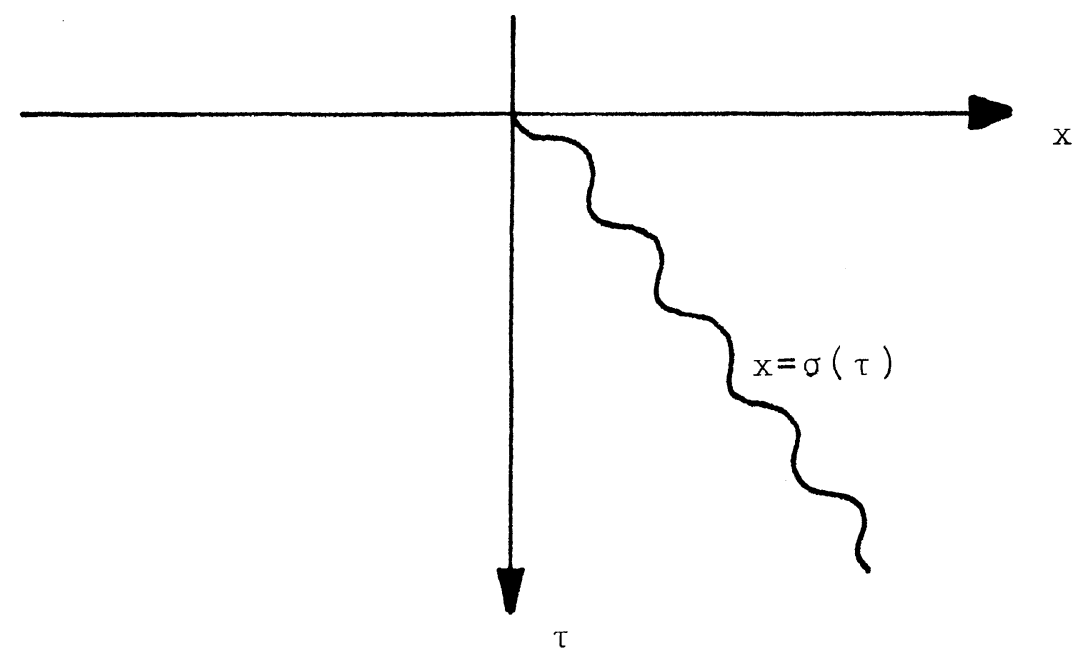

\section{F'ig. 5}

can only freeze and the boundary $\boldsymbol{\sigma}(\tau)$ must be decreasing. This can be substantiated using the strong maximum principle.

\section{Some Further Examples.}

3.1. Let $g \equiv k(x) e^{-\beta t}$. That vertical lines $x=$ constant are the only admissible boundaries is clear, because maximizing $E k\left(x+x_{\tau}\right) e^{-\beta(t+\tau)}$ $=e^{-\beta t} E k\left(x+x_{\tau}\right) e^{-\beta \tau}$ over all stopping times is in effect independent of $t$. Consequently, in the continuation region $\hat{g}$ is to be a linear combination of the functions $\exp ( \pm x \sqrt{2 \beta}-\beta t)$. In view of Theorem $\mathrm{I}$ the problem of finding the optimal reward $\hat{g}$ reduces to finding one or several vertical strips $C$ outside of which $H \leqq 0$ and a linear combination of $\exp ( \pm x \sqrt{2 \beta}-\beta t)$ exceeding $g$ and fitting smoothly with $g$ on the sides of $C$. Then, according to Theorem I, $\hat{g}$ equals this linear combination in $C$ and equals $g$ outside $C$.

For conversation's sake, let us specialize to the case where

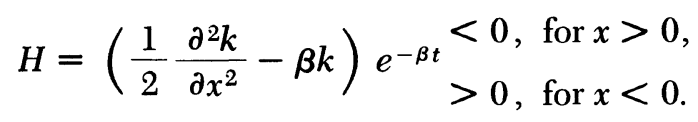

Then the optimal strategy is either to keep playing for ever, or to play in the region $x<x_{0}$ until first hitting $x_{0}$, where $x=x_{0}$ is the unique solution (in the region $H<0$ ) to the equation 


$$
\frac{d}{d x} \log k(x)=\sqrt{2 \beta}
$$

in the latter case

$$
\begin{aligned}
\hat{g} & =k\left(x_{0}\right) \exp \left[\left(x-x_{0}\right)(2 \beta)^{1 / 2}-\beta t\right], \quad \text { for } x<x_{0}, \\
& =k(x) e^{-\beta t}, \quad \text { for } x \geqq x_{0} .
\end{aligned}
$$

3.2 Let $g=t^{-\beta / 2} k(x / \sqrt{t})$. We shall solve this problem by considering first the reward function

$$
f(y, s) \equiv e^{-\beta s} k(y),
$$

for the Ornstein-Uhlenbeck process with generator

$$
A=\frac{1}{2} \frac{\partial^{2}}{\partial y^{2}}-y \frac{\partial}{\partial y} \text {. }
$$

The same discussion holds as in $\S 3.1$ : the optimal boundary consists of one or several vertical lines; in the continuation region $\hat{g}$ must be a linear combination of the functions

$$
g_{\beta} \pm=\int_{0}^{\infty} \exp \left[ \pm \lambda y-\lambda^{2} / 2\right] \lambda^{\beta-1} d \lambda,
$$

which are respectively the increasing and decreasing solutions of the equation $A g=\beta g$. The transformation

$$
x=y e^{s}, \quad t=e^{2 s},
$$

maps the Ornstein-Uhlenbeck process into brownian motion, the reward function (10) into the one announced under $\$ 3.2$ and the vertical boundary $y=\alpha$ into the parabola $x=\alpha \sqrt{t}$ Hence the function under $\$ 3.2$ permits only parabolic optimal boundaries.

As in 3.1, consider the case where $H<0$ for $x>0$ and $H>0$ for $x<0$; then we have the same dichotomy: either the boundary does not exist and playing for ever is best, or the boundary is a parabola $x=\alpha \sqrt{t}$, where $y=\alpha$ is the unique solution (in the region $H<0$ ) to the transcendental equation

$$
\frac{d}{d y} \log \frac{k(y)}{g_{\beta}+(y)}=0 .
$$

Let us further specialize to Dvoretzky's problem $g=x / t$ (cf. $\$ 1$ ), which has the shape given under $\$ 3.2$. Its optimal boundary is a parabola $x=\alpha \sqrt{t}$, where $\alpha$ satisfies the equation (11), which simplifies to Shepp's transcendental equation

$$
\alpha=\left(1-\alpha^{2}\right) \int_{0}^{\infty} \exp \left[\lambda \alpha-\lambda^{2} / 2\right] d \lambda .
$$


Moreover

$$
\begin{aligned}
\hat{g}=\sup _{\tau} E \frac{x+x_{\tau}}{t+\tau} & =\left(1-\alpha^{2}\right) \int_{0}^{\infty} \exp \left[\lambda x-\lambda^{2} t / 2\right] d \lambda, & x<\alpha \sqrt{t}, \\
& =\frac{x}{t}, & x \geqq \alpha \sqrt{t} .
\end{aligned}
$$

At this point, everyone would be tempted to try using the same recipe for other diffusions; namely, take such a stationary reward for an arbitrary diffusion and map it back into brownian motion. You may expect in this way to obtain new shapes for the optimal boundary. But to our regret, the only time-independent diffusions which can be mapped into brownian motion using a space-time deterministic transformation are the Ornstein-Uhlenbeck process and their scale changes. This follows from a result by Cherkasov [10]. Therefore, this method will never give anything more but parabolas or vertical boundaries.

3.3 Some Parabolic Games with Finite Horizon. Consider a reward function of the type

$$
\begin{aligned}
g(x, t) & =(T-t)^{\beta / 2} \varphi\left(\frac{x}{\sqrt{T-t}}\right), t<T, \\
& =C_{1}|x|^{\beta}, \quad x<0, t=T, \\
& =C_{2} x^{\beta}, \quad x \geqq 0, t=T,
\end{aligned}
$$

where $\varphi$ is positive and sufficiently differentiable. Moreover, you must assume that

$$
\lim _{t>T}(T-t)^{\beta / 2} \varphi\left(\frac{x}{\sqrt{T-t}}\right),
$$

exists, in which case it will take on the form $C_{1}{ }^{\prime}|x|^{\beta}$ for $x<0$ and $C_{2}{ }^{\prime} x^{\beta}$ for $x \geqq 0$, with $C_{i} \geqq C_{i}{ }^{\prime} \geqq 0$. Then, as a consequence of the brownian scaling property, $g$ can be shown to have only parabolic optimal boundaries $x=\alpha \sqrt{T-t}$.

Assume $s(t)$ consists of just one parabola $\alpha \sqrt{T-t}$ with the continuation region to the left of it; for short, call

$$
\begin{aligned}
& D(y)=\frac{1}{2}\left[\frac{\partial^{2} \varphi}{\partial y^{2}}+y \frac{\partial \varphi}{\partial y}-\beta \varphi\right], \\
& E(y)=-\frac{\beta-2}{2} D(y)+\frac{y}{2} \frac{\partial D}{\partial y}(y), \\
& F(y)=\frac{1}{2}\left[\frac{\partial^{2} \varphi}{\partial y^{2}}+y \frac{\partial \varphi}{\partial y}-\beta \varphi+\left(C_{1}-C_{1}{ }^{\prime}\right) \beta(\beta-1)(-y)^{\beta-2}\right] .
\end{aligned}
$$


Then $\boldsymbol{\alpha}$ satisfies the transcendental equation

$$
\begin{aligned}
\frac{\alpha D(\alpha)}{2}= & 4 \alpha E(\alpha) \int_{0}^{\alpha} \frac{1}{\sqrt{2 \pi}}\left[\frac{\alpha^{2}-\omega^{2}}{\alpha^{2}+\omega^{2}}\right]^{\beta-2} \exp \left[-\omega^{2} / 2\right] \frac{d \omega}{\alpha^{2}-\omega^{2}} \\
& -\alpha D(\alpha) \int_{0}^{\alpha} \frac{1}{\sqrt{2 \pi}}\left[\frac{\alpha^{2}-\omega^{2}}{\alpha^{2}+\omega^{2}}\right]^{\beta-2} \exp \left[-\omega^{2} / 2\right] d \omega \\
& +\int_{-\infty}^{-\alpha} \frac{1}{\sqrt{2 \pi}} \exp \left[-\eta^{2} / 2\right] \frac{\partial F}{\partial y}(\alpha+\eta) d \eta
\end{aligned}
$$

This equation is obtained by substituting $\alpha \sqrt{T-t}$ for $s(t)$ in equation (9) and by making a couple of changes in variables.

Let us now consider some special cases.

(a) Take a game where you pay one dollar per unit time, as long as you play. You are never rewarded, except at the end where you receive $k x^{2}$ if $x \leqq 0$ and nothing otherwise, with $k>0$. Here

$$
\begin{array}{rlrl}
g & =T-t, & t<T, \\
& =k x^{2}, & & t=T, x \leqq 0, \\
& =0, & & t=T, x \geqq 0 .
\end{array}
$$

This game has the shape under $\$ 3.3$. Hence, the optimal boundary is a parabola $x=\alpha \sqrt{T-t}$, with $\alpha$ satisfying the transcendental equation

$$
\alpha \int_{0}^{\infty} \exp \left[\lambda \alpha-\lambda^{2} / 2\right] d \lambda=k-1 .
$$

(b) In a gambling machine, a device initially at zero position jumps one step to the left or to the right with probability $1 / 2$ after pulling the arm; the gambler wins one dollar each time the device is to the left of its equilibrium position and he loses one dollar otherwise. The maximum number of times $N$ he wishes to play is optional, but it must be decided in advance. For each $N$, how much entrance fee is the casino going to charge in order to break even (on the average) against a clever gambler?

Let $X_{0}$ be the initial position of the device, which in this example is zero but which may be chosen to be any integer in general. Let $X_{i}= \pm 1$ for $i>0$, each with probability $1 / 2$ and let $\epsilon(x)=1$ if $x \leqq 0$ and $=-1$ if $x>0$. The gambler's gain will be

$$
\sum_{n=1}^{p} \epsilon\left(S_{n}\right)
$$


if he decides to play $0 \leqq p \leqq N$ times; agree on putting $\sum_{n=1}^{p}=0$ for $p=0$. The entrance fee to this game equals the maximal expected gain he can achieve, i.e.,

$$
\sup _{0 \leqq p \leqq N} E \sum_{n=1}^{p} \epsilon\left(\mathrm{S}_{n}\right)
$$

Using the markov property and provided $p \geqq 1$,

$$
\begin{aligned}
E \sum_{n=1}^{p} & \epsilon\left(S_{n}\right)=E \epsilon\left(S_{1}\right)+E \sum_{n=2}^{p} \epsilon\left(S_{n}\right) \\
& =\frac{1}{2} \epsilon\left(X_{0}+1\right)+\frac{1}{2} E \sum_{n=1}^{p-1} \epsilon\left(S_{n}{ }^{\prime}\right)+\frac{1}{2} \epsilon\left(X_{0}-1\right)+\frac{1}{2} E \sum_{n=1}^{p-1} \epsilon\left(S_{n}{ }^{\prime \prime}\right),
\end{aligned}
$$

where $S_{n}{ }^{\prime}=\left(X_{0}+1\right)+\sum_{i=1}^{n} X_{i} \quad$ and $\quad S_{n}{ }^{\prime \prime}=X_{0}-1+\sum_{i=1}^{n} X_{i}$. Therefore

$$
\begin{aligned}
\sup _{0 \leqq p \leqq N} E \sum_{n=1}^{p} \epsilon\left(S_{n}\right)=\frac{1}{2}\left[\epsilon\left(X_{0}+1\right)+\epsilon\left(X_{0}-1\right)\right. \\
\left.+\sup _{0 \leqq p \leqq N-1} E \sum_{n: 1}^{p} \epsilon\left(S_{n}{ }^{\prime}\right)+\sup _{0 \leqq p \leqq N-1} E \sum_{n: 1}^{p} \epsilon\left(S_{n}{ }^{\prime \prime}\right)\right]^{+},
\end{aligned}
$$

where $A^{+}=\max (A, 0)$. This simple recurrence relation expresses the entrance fee for the option $N$ in terms of the entrance fee for the option $N-1$. The entrance fees are given by Table 1. In this example the optimal stopping curve as a function of $N$ is given by the largest integer $S_{0}$ with zero entrance fee.

The continuous analogue of this game is one where the gambler pays the amount of time the brownian motion spends in the right half plane and earns the amount of time it spends in the left half plane (cf. Figure 6). How is he going to maximize his gain, if his only choice is between playing until time $T$ or stopping before? A naive gambler would stop as soon as he enters the lose region. But it may pay to lose a bit now in order to win in a more remote future. The problem amounts to maximizing

$$
E \int_{0}^{\tau} H\left(x+x_{s}, t+s\right) d s
$$

over all stopping times $\tau \leqq T-t$, where

$$
\begin{aligned}
H & =-1, & & x>0, \\
& =1, & & x \leqq 0 .
\end{aligned}
$$




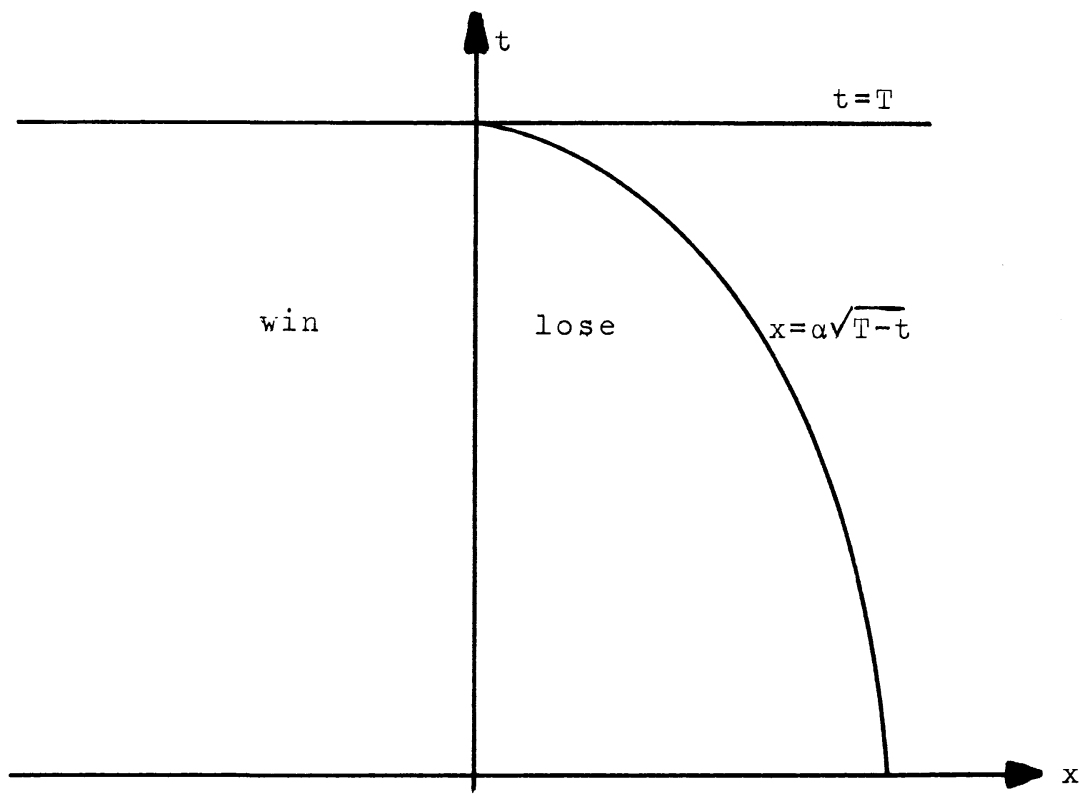

Fig. 6

In view of the result in $\$ 2.4$, this problem can be reduced to a game with reward

$$
\begin{array}{rlrl}
g^{*} & =T-t, & & x>0, t \leqq T, \\
& =T-t+2 x^{2}, & x \leqq 0, t \leqq T .
\end{array}
$$

A simple argument shows that $\hat{g}^{*}=\hat{g}$, where $g$ is the game under (a) with $k=2$. Hence the optimal policy is to stop as soon as the brownian motion enters the region $x \geqq \alpha \sqrt{T-t}$, where $\alpha$ satisfies

$$
\alpha \int_{0}^{\infty} \exp \left[\lambda \alpha-\lambda^{2} / 2\right] d \lambda=1 .
$$

(c) Consider a long, well-insulated tube with water on one side at initial temperature $k-1$ and ice on the other at zero temperature as in Figure 7. With the aid of $\$ 2.8$, it is elementary to check that this is exactly the Stefan problem corresponding to (a). Hence the interface between water and ice describes a parabola $x=\alpha \sqrt{\tau}$ with the parameter $\alpha$ satisfying (13). If $k-1>0$, then $\alpha>0$ and the ice melts; if $k-1<0$, then $\alpha<0$ and the water freezes, because of being supercooled.

It is interesting to observe from (13) that when the initial temperature $k-1$ decreases to -1 , the parameter $\alpha$ tends to $-\infty$, i.e., the 


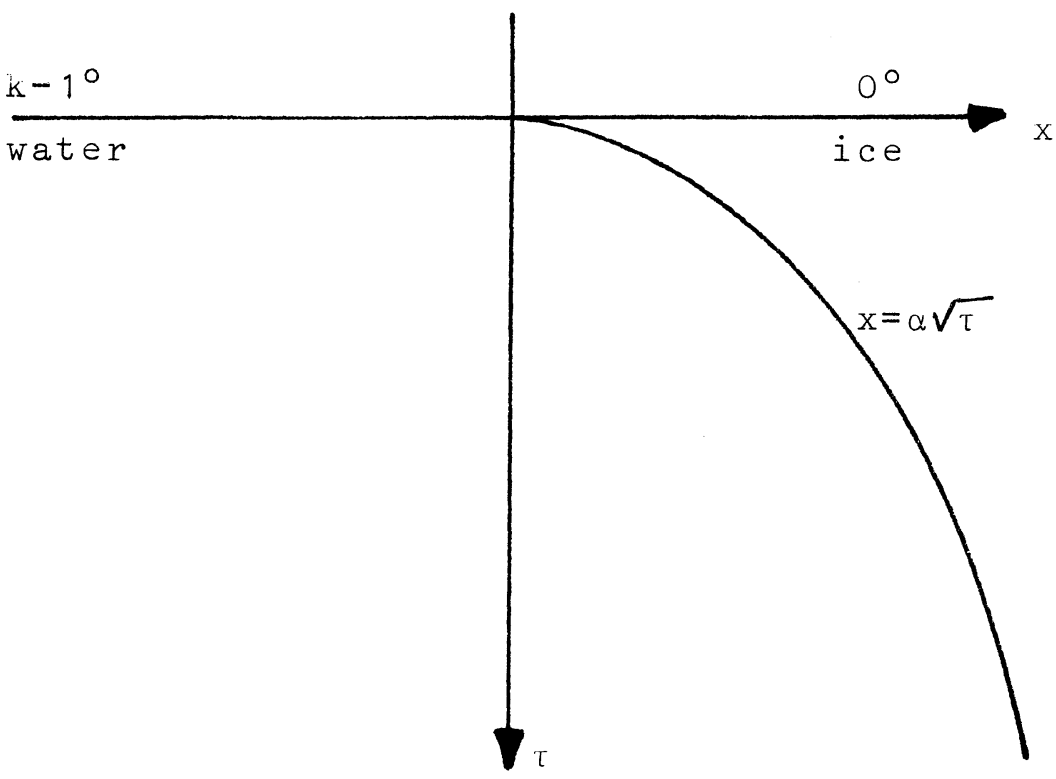

\section{Fig. 7}

liquid phase will freeze faster and faster. One can extrapolate that at temperature -1 , the water freezes instantaneously.

Each of these settings (a), (b), and (c) shows the three different aspects which optimal stopping problems present.

3.4 About the Warrant Pricing Problem. As you recall from $\$ 1$, a warrant is a contract conferring the right to purchase a common stock at a prescribed unit price at any time prior to some expiration date $T$. Samuelson's model for the stock price fluctuations is a logarithmic brownian motion $e^{\sigma x(t)+\delta t}$, where $x(t)$ denotes the ordinary brownian motion starting at $x(0)=0$ and $\sigma$ and $\delta$ are positive constants. Its generator is given by

$$
\frac{\partial}{\partial t}+\frac{\sigma^{2}}{2} y^{2} \frac{\partial^{2}}{\partial y^{2}}+\left(\frac{\sigma^{2}}{2}+\delta\right) y \frac{\partial}{\partial y},
$$

and the expectation of $y(t)$ by

$$
E y(t)=\exp \left[\left(\sigma^{2} / 2+\delta\right) t\right],
$$

where $\sigma^{2} / 2+\delta$ measures the appreciation rate of the stock. Moreover, the warrant appreciates at a rate $\beta$. Also fix the unit price at $y=1$. 
If the warrant holder decides to purchase the common stock at time $\tau$ not exceeding the warrant's lifetime $T$ (which may be infinite) and if the current price is $y$, then his expected gain relative to the unit price will be

$$
E_{y=1} e^{\beta(T-\tau)}\left(y_{\tau}-1\right)^{+}, 0 \leqq \tau \leqq T .
$$

Hence the actual or "rational" price of the warrant is given by

$$
\sup _{0 \leqq \tau \leqq T} E_{y=1} e^{\beta(T-\tau)}\left(y_{\tau}-1\right)^{+} .
$$

So $g(y, t)=e^{\beta(T-t)}(y-1)^{+}$is the reward function and $\hat{g}$, given by (14) satisfies

$$
\left(\frac{\partial}{\partial t}+\frac{\sigma^{2}}{2} y^{2} \frac{\partial^{2}}{\partial y^{2}}+\left(\frac{\sigma^{2}}{2}+\delta\right) y \frac{\partial}{\partial y}\right) \hat{g}=0,
$$

in the continuation region, together with the final condition

$$
\hat{\mathrm{g}}(y, T)=(y-1)^{+},
$$

and the two smooth fit relations at the boundary $r(t)$ of the continuation region. In the case of an infinite horizon the final condition is absent.

It is obvious that the optimal boundary must belong to the region where $g>0$, i.e., where $y>1$; in the latter region

$$
\begin{aligned}
H \equiv & \left(\frac{\partial}{\partial t}+\frac{\sigma^{2}}{2} y^{2} \frac{\partial^{2}}{\partial y^{2}}+\left(\frac{\sigma^{2}}{2}+\delta\right) y \frac{\partial}{\partial y}\right) g, \\
& =e^{-\beta t}\left(\left(\delta+\frac{\sigma^{2}}{2}-\beta\right) y+\beta\right) .
\end{aligned}
$$

Unless the appreciation rate $\beta$ of the warrant exceeds the appreciation rate $\alpha=\sigma^{2} / 2+\delta$ of the stock, the solution to the problem is trivial, because then $H \geqq 0$ in the region $y>1$ : the optimal strategy is to wait as long as you can before purchasing the stock. Then if $T=$ $\infty, \hat{g}=\infty$ and, if $T<\infty$,

$$
\begin{aligned}
\hat{g}=\frac{1}{\sigma} \int_{1}^{\infty} \frac{1}{\sqrt{2 \pi(T-t)}} \exp & {\left[-\frac{1}{2(T-t)}\left(\frac{\delta}{\sigma}(T-t)\right.\right.} \\
+ & \left.\left.\frac{1}{\sigma} \log \frac{y}{z}\right)^{2}\right] \frac{(z-1)}{z} d z .
\end{aligned}
$$

So the interesting case is $\beta>\alpha$. Consider first an infinite horizon $T=\infty$. The translation invariance of the reward function $g$ (up to an exponential $e^{-\beta t}$ ) in the vertical direction suggests that the optimal 
boundary must be time-independent. This fact combined with the smooth fit condition shows that to maximize your gain you must wait until the current price of the stock exceeds

$$
y_{1}=\frac{\left(\frac{\delta^{2}}{\sigma^{2}}+2 \beta\right)^{1 / 2}-\frac{\delta}{\sigma}}{\left(\frac{\delta^{2}}{\sigma^{2}}+2 \beta\right)^{1 / 2}-\frac{\delta}{\sigma}-\sigma},
$$

and to the left of $y_{1}$, the optimal reward equals

$$
C e^{-\beta t} y^{1 / \sigma\left(\sqrt{\delta^{2 / \sigma}+2 \beta}-\delta / \sigma\right)},
$$

where $C$ is an appropriate constant expressed in terms of $\beta, \delta$ and $\sigma$. For future use, let $\hat{g}_{\infty}$ stand for (15).

However, for a finite warrant, the optimal buying curve depends explicitly on the time. Moreover, the optimal boundary $r(t)$ belongs to the vertical strip between

$$
y_{0} \equiv \frac{\beta}{\beta-\delta-\sigma^{2} / 2} \text { and } y_{1}=\frac{\left(\frac{\delta^{2}}{\sigma^{2}}+2 \beta\right)^{1 / 2}-\frac{\delta}{\sigma}}{\left(\frac{\delta^{2}}{\sigma^{2}}+2 \beta\right)^{1 / 2}-\frac{\delta}{\sigma}-\sigma}
$$

because on the one hand it must belong to the region where $H \leqq 0$, i.e., where $y \geqq y_{0}$ and on the other hand to the region where $g<\hat{g}_{\infty}$, i.e., where $y<y_{1}$. The boundary $r(t)(t<T)$ can be shown to be decreasing and continuously differentiable; see Figure 8 . Also near the date of expiration $T, r(t)$ behaves like

$$
y_{0}+\alpha \sqrt{T-t}
$$

where $\alpha$ satisfies the transcendental equation

$$
\begin{aligned}
\frac{\alpha^{2}}{2}= & -\alpha^{2} \int_{0}^{\alpha} \frac{1}{\sqrt{2 \pi}} \frac{\alpha^{2}-\omega^{2}}{\alpha^{2}+\omega^{2}} \exp \left[-\omega^{2} / 2\right] d \omega \\
& +\int_{-\infty}^{-\alpha} \frac{1}{\sqrt{2 \pi}} \exp \left[-\eta^{2} / 2\right] d \eta .
\end{aligned}
$$

Finally, when $T$ tends to infinity, i.e., when you have plenty of time left before the warrant expires, $r(t)$ approaches $y_{1}$ at an exponential rate (for fixed $t$ ). This is done by comparing $r(t)$ with the optimal boundary for a game where the only other strategy besides quitting is to continue the game until hitting the horizon or the line $y=y_{1}$. 


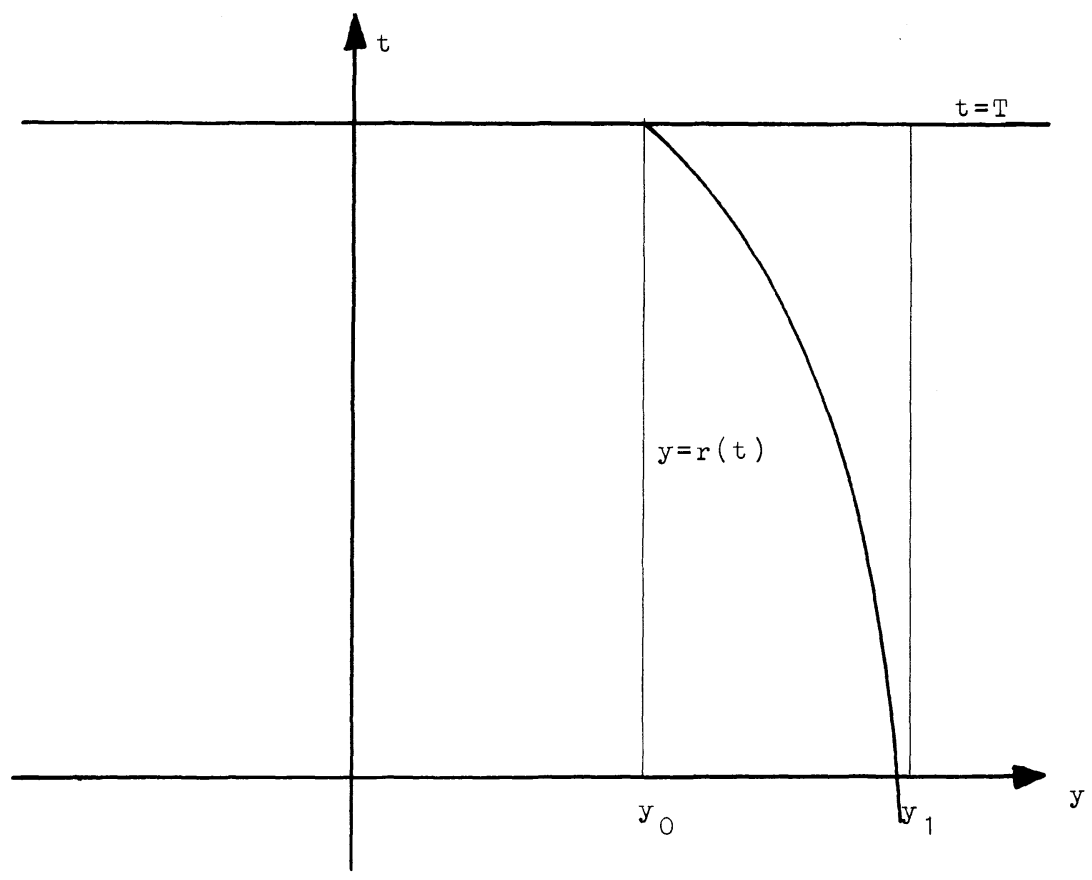

Fig. 8

3.5 A Bond-Selling Problem. W. M. Boyce [6] has considered the problem when to sell "Brownian" bonds. The price of a bond converges to its face value near maturity, which is achieved by imposing an appropriate drift on the brownian price fluctuations. When is the owner going to sell his bonds in order to have the maximal capital gain? For tax or other reasons the owner may want to sell before expiration, i.e., before a certain date $T$ prior to the expiration date. Here again we ask how to sell optimally. In the latter case the problem is not well posed, unless some information is available about the price distribution at the ultimate selling date $T$. But the experienced investor may be able to guess or predict the price's mean $m$ and variance $\sigma^{2}$ at that moment. In fact, the most random distribution fitting these requirements is a normal distribution $N\left(m, \sigma^{2}\right)$.

In both problems, agree to choose $T=1$. H. Fölmer [23] shows that a diffusion $y_{s}$ starting at $y_{0}=y$ constrained to have a normal distribution $N\left(m, \sigma^{2}\right)$ at time $s=1$ is governed by

$$
\frac{\partial}{\partial s}+\frac{1}{2} \frac{\partial^{2}}{\partial y^{2}}+(y a(s)+m b(s)) \frac{\partial}{\partial y}, s \leqq 1,
$$


where

$$
a(s)=\frac{\sigma^{2}-1}{1+s\left(\sigma^{2}-1\right)} \text { and } b(s)=\frac{1}{1+s\left(\sigma^{2}-1\right)} .
$$

This includes both problems, because for the first one $m$ is the face value and $\sigma=0$. Without loss of generality you may assume $m=0$. The reward function is $g(y, s)=y$, because $y$ is the gain you make by selling the bond at price $y$. consequently, the problem amounts to maximizing

$$
E_{y, s}\left(y_{\tau}\right) \text {, }
$$

over all stopping times not exceeding $1-s$. The payoff-rate

$$
H=\frac{y\left(\sigma^{2}-1\right)}{1+s\left(\sigma^{2}-1\right)}
$$

vanishes for $\sigma=1$, is negative for $y>0$ when $\sigma<1$ and positive for $y<0$ when $\sigma>1$. The fact that for $\sigma<1, H$ is negative in $x>0$ implies that the continuation region will be unbounded to the left and bounded to the right. For $\sigma>1$, the situation reverses completely. Of course for $\boldsymbol{\sigma}=1$, the problem is vacuous because then $g$ itself is parabolic with respect to the generator (17). Notice that for $\sigma=0$,

$$
r(s)=\alpha \sqrt{1-s},
$$

where $\alpha$ satisfies the transcendental equation (12). This is easily seen from the transformation

$$
1-\left(\sigma^{2}-1\right) t=\frac{1}{1+s\left(\sigma^{2}-1\right)}
$$

and

$$
x=\frac{y}{1+s\left(\sigma^{2}-1\right)},
$$

which maps the motion governed by (17) into brownian motion and the reward function $g$ into

$$
\frac{x}{1-\left(\sigma^{2}-1\right) t}, \text { for } t \leqq \frac{1}{\sigma^{2}} .
$$

For $\boldsymbol{\sigma}>0, \boldsymbol{\sigma} \neq 1$, the boundary can be shown to behave approximately like

$$
y=\alpha(1-s)^{1 / 2},
$$


for small $1-s$, where $\alpha>0$ (for $1>\sigma>0$ ) and $-\alpha>0$ (for $\sigma>1$ ) satisfy the transcendental equation (16). Boyce's numerical calculations show that the optimal boundary is the farthest to the right for $\sigma=0$, that it decreases to a non-trivial limiting curve when $\sigma \nearrow 1$, that it jumps to the quadrant $y<0$ when $\sigma$ passes through 1 and that it increases thereafter; see Figure 9. D. S. Griffeath and J. L. Snell [25] have obtained a similar result with the discrete version of the problem. This phenomenon can be proved by observing that the optimal boundary for the reward function

$$
g(y, s)=y \times \operatorname{sign} \text { of }(1-\sigma),
$$

decreases with respect to $\sigma$; a continuity argument does the rest.

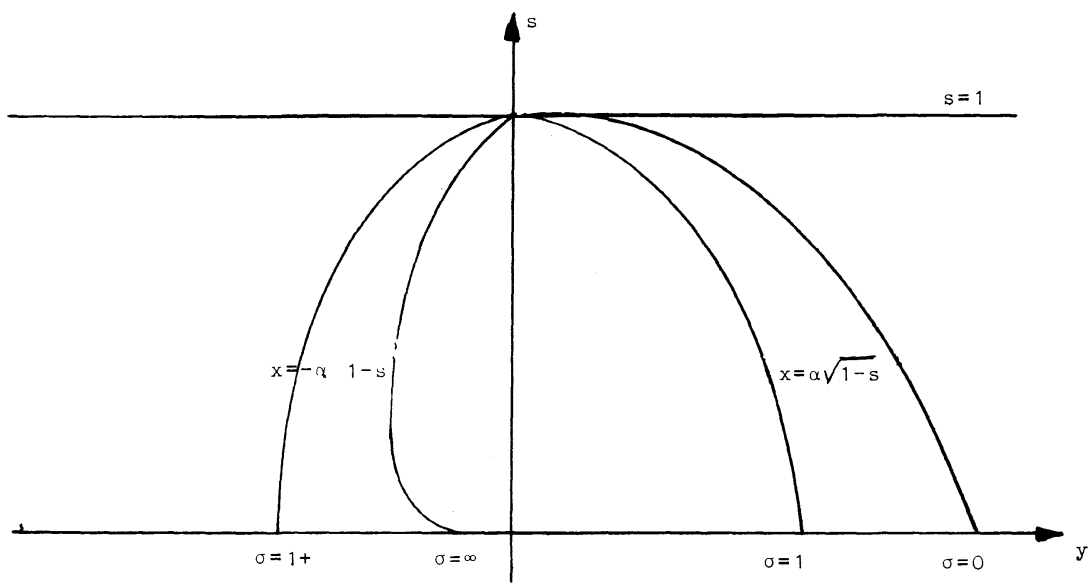

3.6 About holes in the Continuation region. In this section, we provide an example, showing how a small continuous modification will suffice to generate a hole in the continuation region. In fact, this procedure can be generalized so as to get as many holes as you wish.

(1) Consider the following game

$$
\begin{aligned}
g & =(T-t)\left(a+3 \log \frac{1}{T-t}\right), \quad t<T, \\
& =\text { a delta-function with pole at zero, } t=T .
\end{aligned}
$$

Its optimal reward is explicitly computable (cf. Figure 10); indeed,

(18) $\hat{g}=A\left(x^{2}+(T-t)\right)+\frac{1}{\sqrt{2 \pi(T-t)}} \exp \left[-x^{2} / 2(T-t)\right]$, 
in the continuation region $-s(t)<x<s(t)$, where

$$
s(t)=\left(2(T-t) \log \frac{A^{-1}}{2 \sqrt{2 \pi}(T-t)^{3 / 2}}\right)^{1 / 2} ;
$$

$A$ is chosen such that

$$
3+2 \log \frac{A^{-1}}{2 \sqrt{2 \pi}}=a
$$

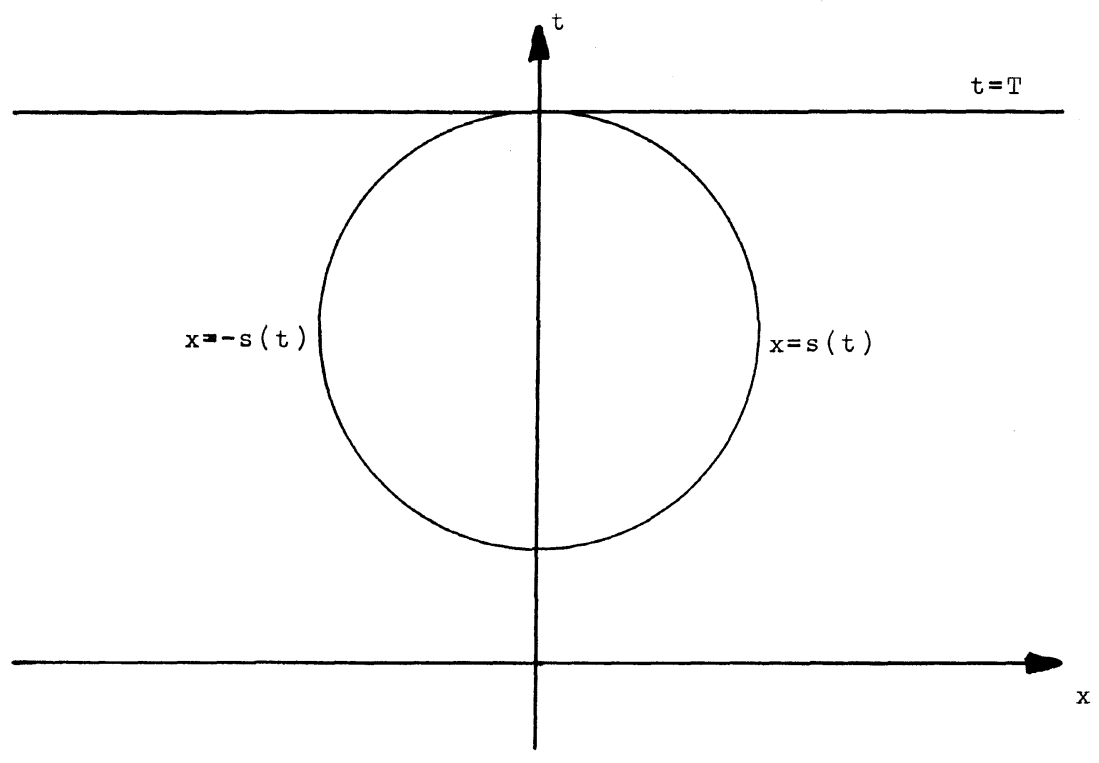

Fig. 10

These expressions are derived from considering a one-dimensional heat flow problem with initial temperature $u(x, 0) \equiv A x^{2}+$ a delta function centered at zero; then its temperature at time $T-t$ equals

$$
u(x, t)=A\left(x^{2}+(T-t)\right)+\frac{1}{\sqrt{2 \pi(T-t)}} \exp \left[-x^{2} / 2(T-t)\right] ;
$$

but $u$ as a function of $x$ has two minima, which describe in the $(x, t)$ plane the curves $x= \pm s(t)$. So, it satisfies the heat equation and the second smooth-fit relation:

$$
\frac{\partial u}{\partial x}(s(t), t)=0=\frac{\partial g}{\partial x}(s(t), t) .
$$


The other one

$$
u(s(t), t)=g(s(t), t)
$$

determines $A$ as a function of $a$, as expressed in (20). Since $u>g$ in the interval $[-s(t), s(t)]$, Theorem 1 implies (18) and (19).

(2) Let $g$ be as in (1) except that now the final gain $h$ is the sum of two delta-functions with poles respectively at $-a$ and $a$. Choose $a$ to be large. You would expect the continuation region to be composed of two separate blobs, each one rather like the original picture (Figure 10) but slightly distorted due to the presence of the two delta-functions. In fact, this is not so: each of the two connected components is an exact replica of the original continuation region as in Figure 11. The smooth fit relations and Theorem I are the key to this somewhat surprising fact: the function (18) centered at $-a$, resp. $+a$, fits smoothly with $g$ and at $t=T$ it reduces to a delta-function with pole at $-a$, resp. $+a$. So as long as $+a$ is such that the two blobs do not overlap, they remain unchanged.

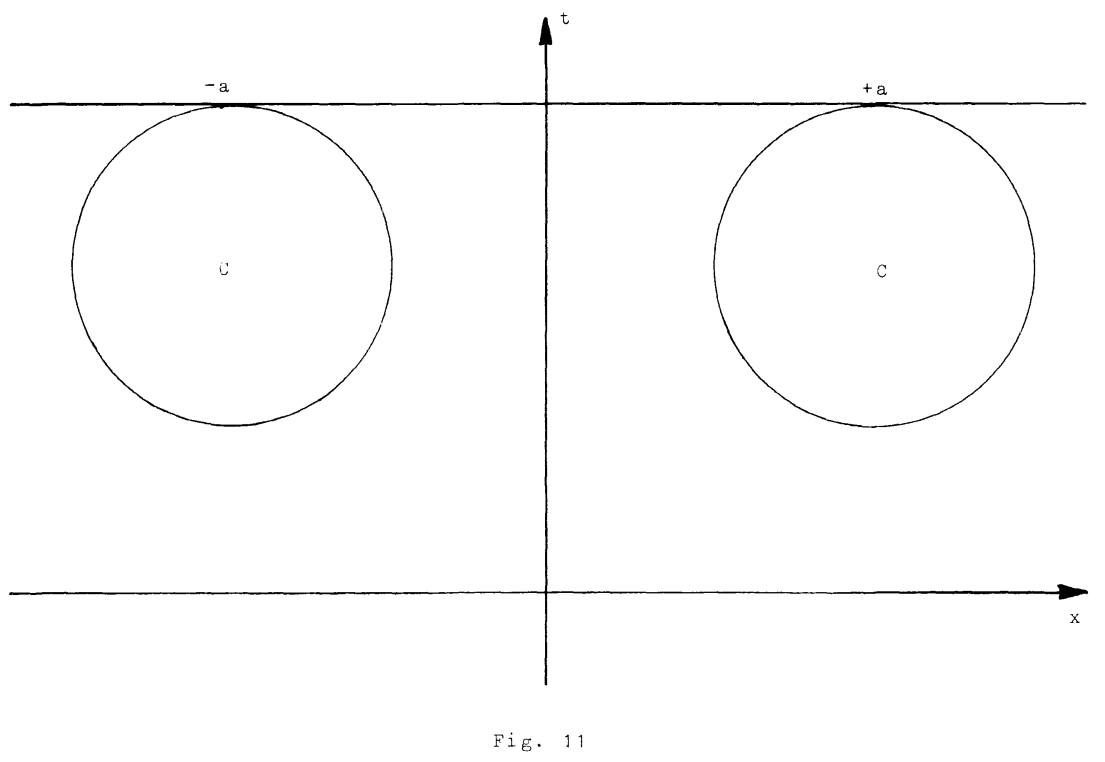

(3) As soon as the blobs overlap so as to be linked, a deformation takes place because the brownian particle can move freely from the one into the other; the picture will look like Figure 12. In fact that part of the continuation region ahead of $t_{1}$ (cf. Figure 12) will remain unchanged irrespective of $a$. 


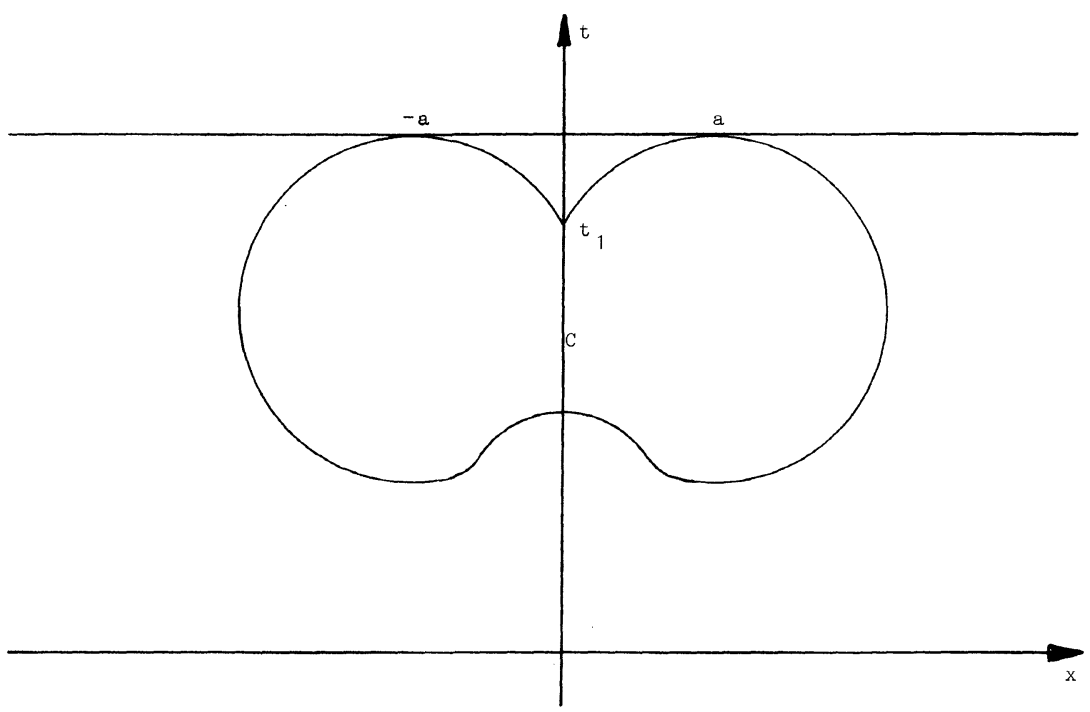

Fig. 12

(4) In the final step, you smoothen $h$ somewhat by replacing it by

$$
\frac{1}{\sqrt{2 \pi \epsilon}} \exp \left[-(x+a)^{2} / 2\right]+\frac{1}{\sqrt{2 \pi \epsilon}} \exp \left[-(x-a)^{2} / 2\right]
$$

with $\epsilon>0$ small. Now $h>0$ everywhere which adds to the picture a narrow strip of continuation points near the horizon. In this fashion you get a hole of stopping points in the interior of the continuation region (cf. Figure 13). By making $a$ small or $\epsilon$ large, the hole will shrink down to a point and finally disappear. Obviously this construction can be repeated so as to get as many holes as you wish.

4. Some Final Remarks. In this final section we will comment on the shape of the continuation region for a reward function of the type

$$
\begin{aligned}
g & =T-t, & & t<T, \\
& =h(x)>0, & t=T, & -a<x<a, \\
& =0, & t & =T, \quad|x| \geqq a,
\end{aligned}
$$

where $h(x)$ is a $C^{3}$-function in $[-a, a]$, subject to the lateral conditions $\partial h / \partial x( \pm a)=0$ and $\frac{1}{2} \partial^{2} h / \partial x^{2}( \pm a)=-H=1$. These assumptions will be in force throughout this section.

THEOREM II. If $\frac{1}{2} \partial^{2} h / \partial x^{2}$ has at most two zeros and $\frac{1}{2} \partial^{2} h / \partial x^{2}-$ 


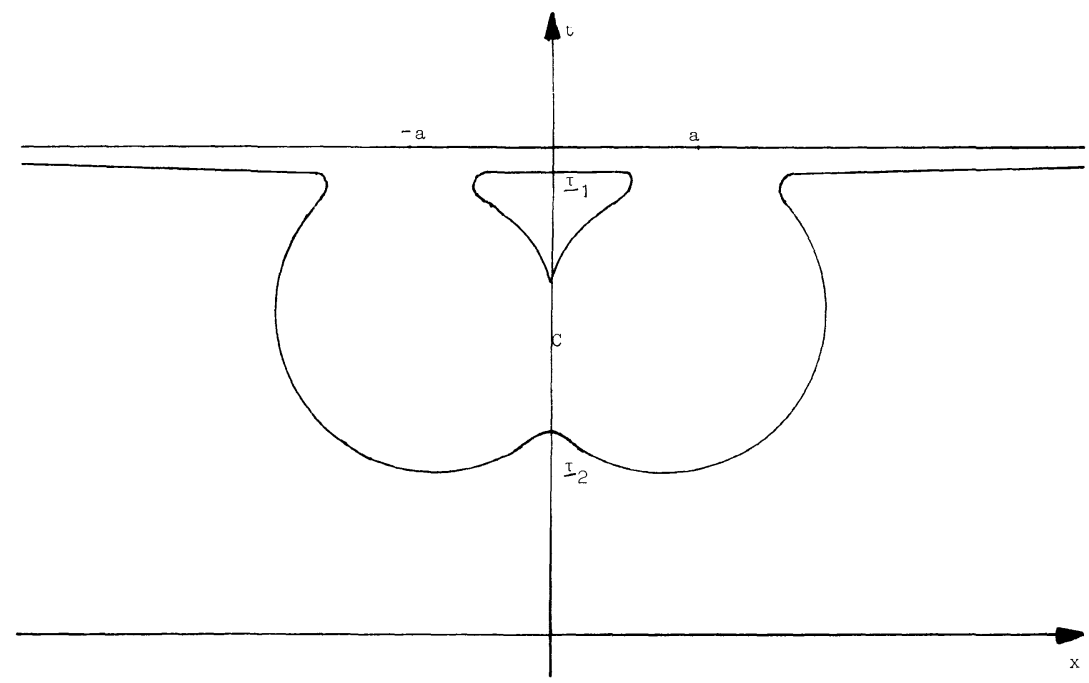

Fig. 13

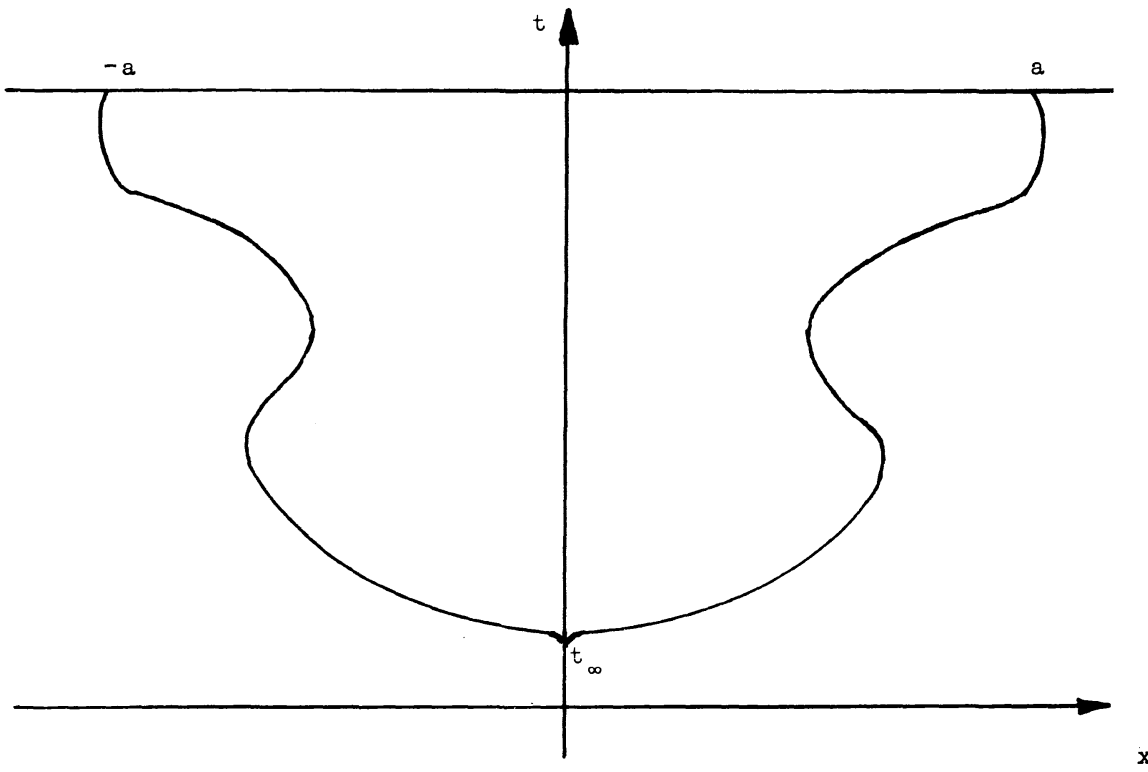

Fig. 14 
1 at most a finite number of zeros in $[-a, a]$, then the continuation region is a connected bounded region, whose boundary $x=s(t)$ is a continuously differentiable function as long as $t \geqq t_{\infty}>-\infty, t_{\infty}$ being the relative minimum of the continuation region; see Fig. 14. Moreover, the number of zeros of $\dot{s}$ cannot exceed the number of zeros of $-\frac{1}{2} \partial^{2} h / \partial x^{2}+1$.

REMARK. The lateral conditions for $h$ at the points $\pm a$ are essential to give the boundary a nice start at $t=T$. Otherwise the boundary may behave in a very singular way near the horizon.

As to the zeros of $\frac{1}{2} \partial^{2} h / \partial x^{2}$, we could have assumed only a finite number of them, in which case this number would have appeared to be an upper bound to the number of critical points.

Outline of The Proof. We shall make the simplifying assumption that $h(x)$ is an even function; then the continuation region is symmetric in $x$. We will now give the main ingredients of the proof.

1. Local Result: Starting from the finite horizon, $\dot{s}$ does not blow up for a little while. This is achieved by proving the existence of a continuously differentiable solution to an integral equation in $s(t)$ (similar to (9)) in a small time interval $(T-\epsilon, T]$, using a fixed point method. This solution turns out to coincide with the optimal boundary in that time-interval. The length of that interval merely depends on

$$
\sup _{|x|<a}\left|\frac{\partial^{3} h}{\partial x^{3}}\right| \text {. }
$$

Subsequently, you extend the boundary down to a point $t_{0}$ where $\dot{s}$ ceases to exist. If $s\left(t_{0}\right)=0$, you would have reached the bottom of $C$ (see Fig. 14) and since $h$ is even, the proof would be finished. So $s\left(t_{0}\right)>0$ is the interesting case. All the effort of Theorem II is put into proving that $s(t)$ can be extended a bit below $t_{0}$ in a continuously differentiable way under the assumption that the boundaries have not yet intersected. This extension is possible provided $|\dot{s}|$ is bounded in $\left(t_{0}, T\right]$; the reason for this will be explained in 2 .

2. Why does the boundedness of $|\dot{s}|$ in $\left(t_{0}, T\right]$ suffice to guarantee its extension beyond $t_{0}$ ? From (8) we deduce that

$$
\frac{\partial^{3} \hat{g}}{\partial x^{3}}(s(t), t)=4 \dot{s}(t) \text {. }
$$

Since $|\dot{s}|$ is bounded and since $\partial^{3} \hat{g} / \partial x^{3}(x, T)=\partial^{3} h / \partial x^{3}$ is also bounded, the maximum principle tells you that $\partial^{3} \hat{g} / \partial x^{3}$ is bounded throughout the continuation region between $t_{0}$ and $T$. Choose an arbitrary horizon $T_{1}$ between $t_{0}$ and $T$ and define 


$$
\begin{aligned}
g_{1}(x, t) & =T_{1}-t, \quad \text { for } t<T_{1}, \\
& =h_{1}(x) \equiv \hat{g}\left(x, T_{1}\right)-g\left(x, T_{1}\right), \quad \text { for } t=T_{1} .
\end{aligned}
$$

It is plain that $g_{1}$ 's optimal boundary denoted by $s_{1}(t)$ is the same as $s(t)$ below the level $T_{1}$. But now 1 . applies to $g_{1}(x, t)$ and it tells you that the optimal boundary is continuously differentiable in a time interval $\left(T_{1}-\epsilon, T_{1}\right]$, whose length depends on

$$
\sup _{|x|<s\left(T_{1}\right)}\left|\frac{\partial^{3} h_{1}}{\partial x^{3}}\right|
$$

the latter is bounded above by

$$
\sup _{\substack{t_{0}<t \leq T \\-s(t) \leqq x \leqq s(t)}}\left|\frac{\partial^{3} \hat{g}}{\partial x^{3}}(x, t)\right|<\infty,
$$

independently of $T_{1}$. Hence, the length $\epsilon$ of the interval is actually independent of $T_{1}$. Therefore, by choosing $T_{1}>t_{0}$ close enough to $t_{0}$, the optimal boundary is seen to be differentiable below $t_{0}$, as advertised.

3. Moreover, $\frac{1}{2} \partial^{2} g / \partial x^{2}$ remains strictly positive in a strip around the boundary. The function $\frac{1}{2} \partial^{2} \hat{g} / \partial x^{2}$ is continuous in $-s(t) \leqq x \leqq$ $s(t)$ for $t_{0}<t \leqq T$ and it satisfies the boundary value problem, defined by

$$
\begin{gathered}
\left(\frac{\partial}{\partial t}+\frac{1}{2} \frac{\partial^{2}}{\partial x^{2}}\right) \frac{1}{2} \frac{\partial^{2} \hat{g}}{\partial x^{2}}=0, \quad-s(t)<x<s(t), t_{0}<t \leqq T, \\
\frac{1}{2} \frac{\partial^{2} \hat{g}}{\partial x^{2}}( \pm s(t), t)=1,
\end{gathered}
$$

and

$$
\frac{1}{2} \frac{\partial^{2} \hat{g}}{\partial x^{2}}(x, T)=\frac{1}{2} \frac{\partial^{2} h}{\partial x^{2}} .
$$

The key to the whole problem is the following conservation law: the total " amount of heat",

$$
\int_{-s(t)}^{s(t)} \frac{1}{2} \frac{\partial^{2} \hat{g}}{\partial x^{2}}(x, t) d x=\frac{1}{2}\left(\frac{\partial \hat{g}}{\partial x}(s(t), t)-\frac{\partial \hat{g}}{\partial x}(-s(t), t)\right)=0
$$

remains zero, because of the smooth fit conditions. This fact combined with the boundary condition (24) implies that there has to be at least one zero and by symmetry exactly two zeros, since by assumption $\partial^{2} h / \partial x^{2}$ has at most two zeros. Hence, the "root" curves 
$\frac{1}{2} \partial^{2} \hat{g} / \partial x^{2}=0$ will extend downwards and they can only intersect the boundary at the very moment all the "heat" has disappeared, i.e., at $t_{\infty}$ (see Figure 15). Otherwise, either (25) or the maximum principle would be violated. As a bonus you find that the continuation region has to close down at the bottom.

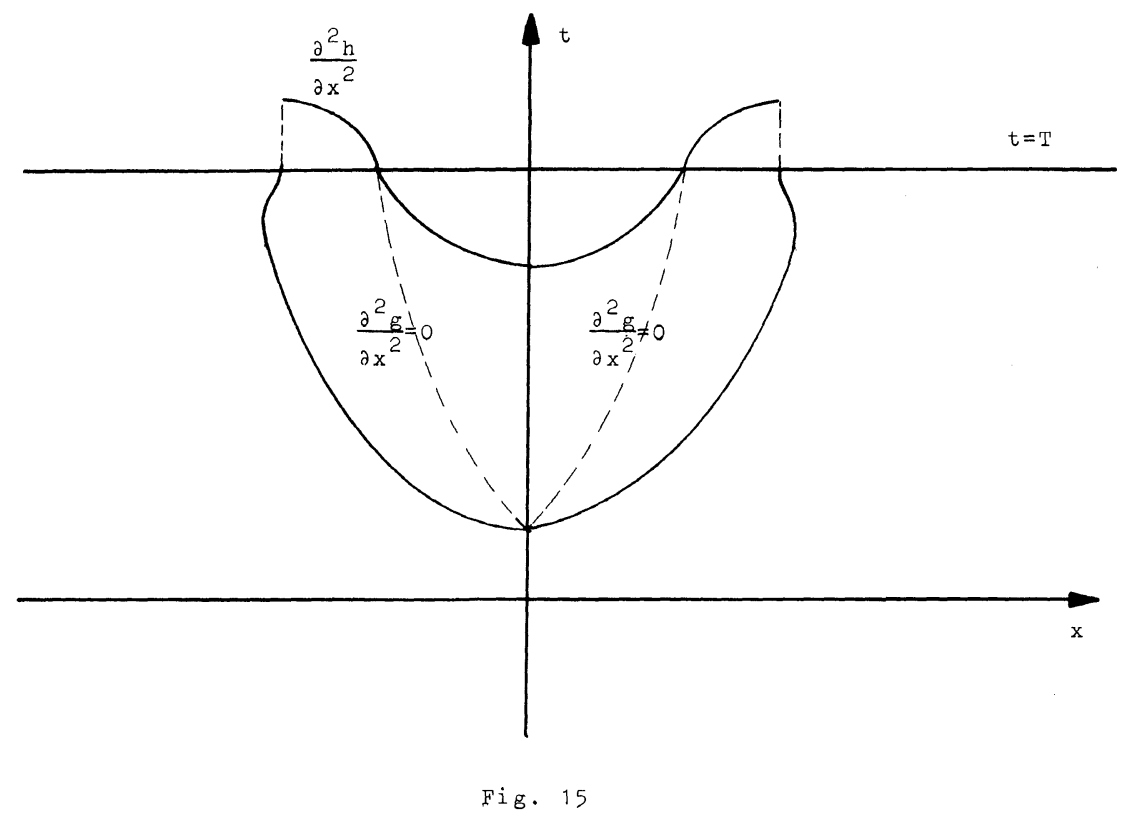

4. The boundary can be extended as long as $\frac{1}{2} \partial^{2} \hat{g} / \partial x^{2}>0$ in a strip around the boundary. The reader familiar with Kac's [28] "principle of not feeling the curvature" knows that a brownian observer close enough to the boundary will feel it only as a straight line, to a first approximation. This idea is now adapted to the space-time brownian motion so that the boundary can actually be replaced by a tangent line near the point under consideration. The estimates, expressing the optimality of $\hat{g}$, performed in this simplified region, provide an upper bound for $\dot{s}$, while the integral equation for $s(t)$ yields a lower bound to $\dot{s}$. The latter estimate relies on the local monotonicity of the boundary; this is so, because the number of zeros of $\dot{s}$ is bounded above by the number of zeros of $\frac{1}{2} \partial^{2} h / \partial x^{2}-1$. This finishes the proof of Theorem II.

In view of the conservation law, cited above, it is of special interest to draw the analogy between the game defined by (22) and the icemelting or ice-freezing problem. As was pointed out in $\$ 2.8$, the 
functions $\quad w(x, \tau)=-\partial / \partial t(\hat{g}-g)(x, T-\tau) \quad$ and $\quad \sigma(\tau)=s(T-\tau)$ satisfy

$$
\begin{aligned}
& \frac{\partial w}{\partial \tau}=\frac{1}{2} \frac{\partial^{2} w}{\partial x^{2}}, \quad \text { in }[-\boldsymbol{\sigma}(\tau), \boldsymbol{\sigma}(\tau)], \\
& w( \pm \boldsymbol{\sigma}(\tau), \tau)=0, \\
& \frac{\partial w}{\partial x}( \pm \boldsymbol{\sigma}(\tau), \tau)=\mp 2 \dot{\boldsymbol{\sigma}}(\tau), \\
& w(x, 0)=\frac{1}{2} \frac{\partial^{2} h}{\partial x^{2}}-1 .
\end{aligned}
$$

The curves $\pm \boldsymbol{\sigma}(\tau)$ represent the position in time of the interface between water and ice, water at temperature $w(x, \tau)$ in the region $|x|<\boldsymbol{\sigma}(\tau)$ and ice at zero temperature in the complementary region. Of course, you permit the temperature of the water to be negative, i.e., supercooled, and positive in different areas. Observe that

$$
\int_{-\sigma(\tau)}^{\sigma(\tau)} w(x, \tau) d x=\int_{-\sigma(\tau)}^{\sigma(\tau)}\left(\frac{1}{2} \frac{\partial^{2} \hat{g}}{\partial x^{2}}-1\right) d x=-2 \sigma(\tau),
$$

and, in particular,

$$
\int_{-a}^{a} w(x, 0) d x=-2 a
$$

this means that at each moment $\tau$, the total amount of heat equals the total amount of heat needed to freeze all the water. That for optimal stopping problems this is valid is an important fact: it is responsible for the compactness of the continuation region and for the fact that the continuation region closes down at the bottom.

It is instructive to observe what occurs when (26) is not valid:

(a) First assume that the total amount of heat would exceed the amount necessary to freeze the liquid:

$$
\int_{-a}^{a} \frac{\partial^{2} h}{\partial x^{2}}>0
$$

i.e., the liquid would not be sufficiently supercooled to become completely solid. Then there always would be a residual amount of unfrozen water and it is intuitively obvious that this residual amount equals the excess amount of heat which would have to be extracted from the system to achieve complete freezing (cf. Figure 16). In view of $\S 4.3$, this case corresponds to the situation where the root curves of 
$\partial^{2} \hat{g} / \partial x^{2}$ meet each other before meeting the boundary. Then the boundary can be extended indefinitely, as a result of the argument in Theorem II.

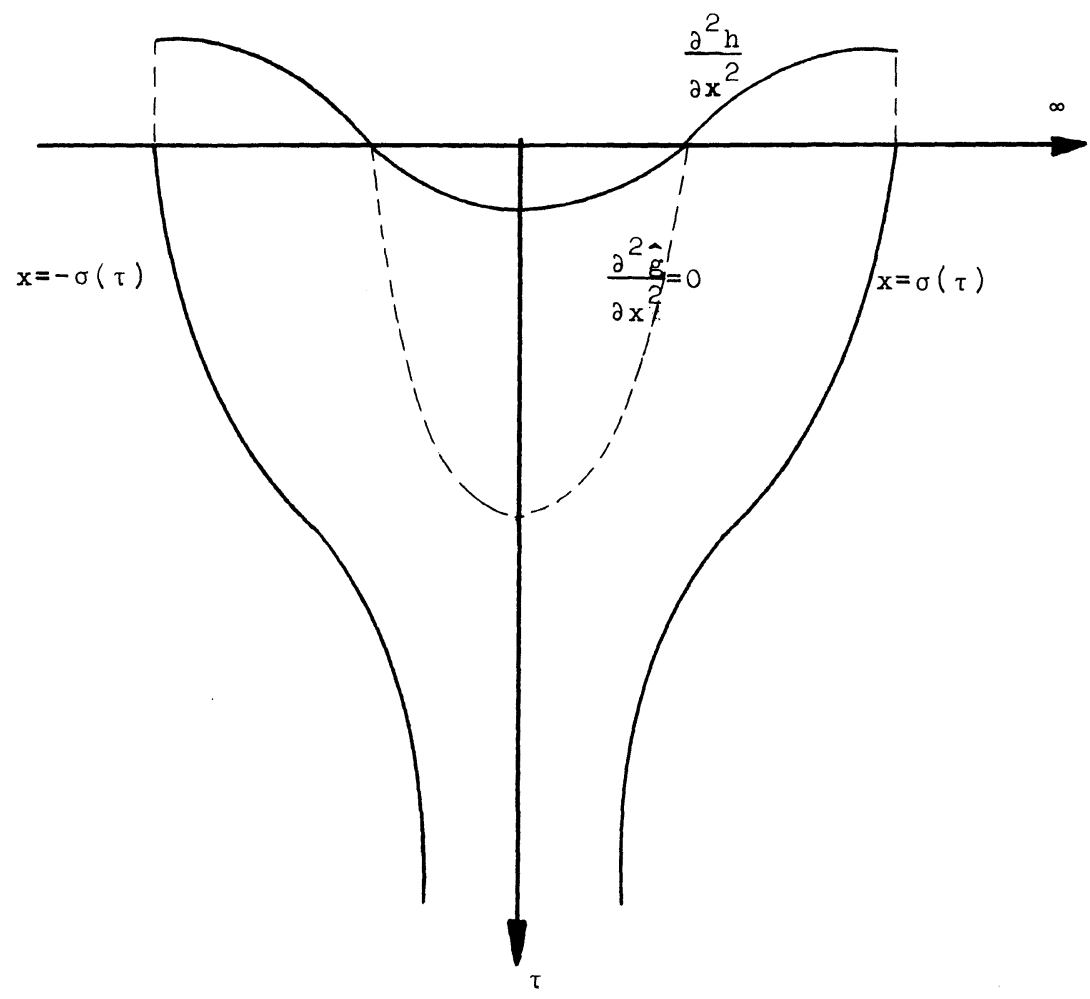

Fig. 16

(b) Next suppose that the total initial amount of heat would be less than the amount necessary to freeze the liquid:

$$
\int_{-a}^{a} \frac{\partial^{2} h}{\partial x^{2}}<0
$$

i.e., the liquid would be too cold. Then one expects a swift freezing of the water. But the smaller the distance between both pieces of ice, the smaller the temperature gets in view of the fact that an inescapable amount of cold (negative heat) will remain. But the maximum principle does not allow the water to decrease its temperature below the minimum of its initial temperature. Therefore at some stage something 
drastic must happen (see Figure 17). But as long as the root curves of $\partial^{2} \hat{\mathrm{g}} / \partial x^{2}$ do not intersect the boundary, the arguments of Theorem II show that the boundary can be extended downwards in a smooth way. At the very moment the root curves of $\partial^{2} \hat{g} / \partial x^{2}$ meet the boundary (as it must, because of (26)), the solution ceases to exist, because the boundary condition

$$
\left.\frac{1}{2} \frac{\partial^{2} \hat{g}}{\partial x^{2}}( \pm \sigma(\tau), \tau)\right),=1,
$$

cannot be satisfied any more; one may think of this as a sudden and instantaneous freezing of all the water.

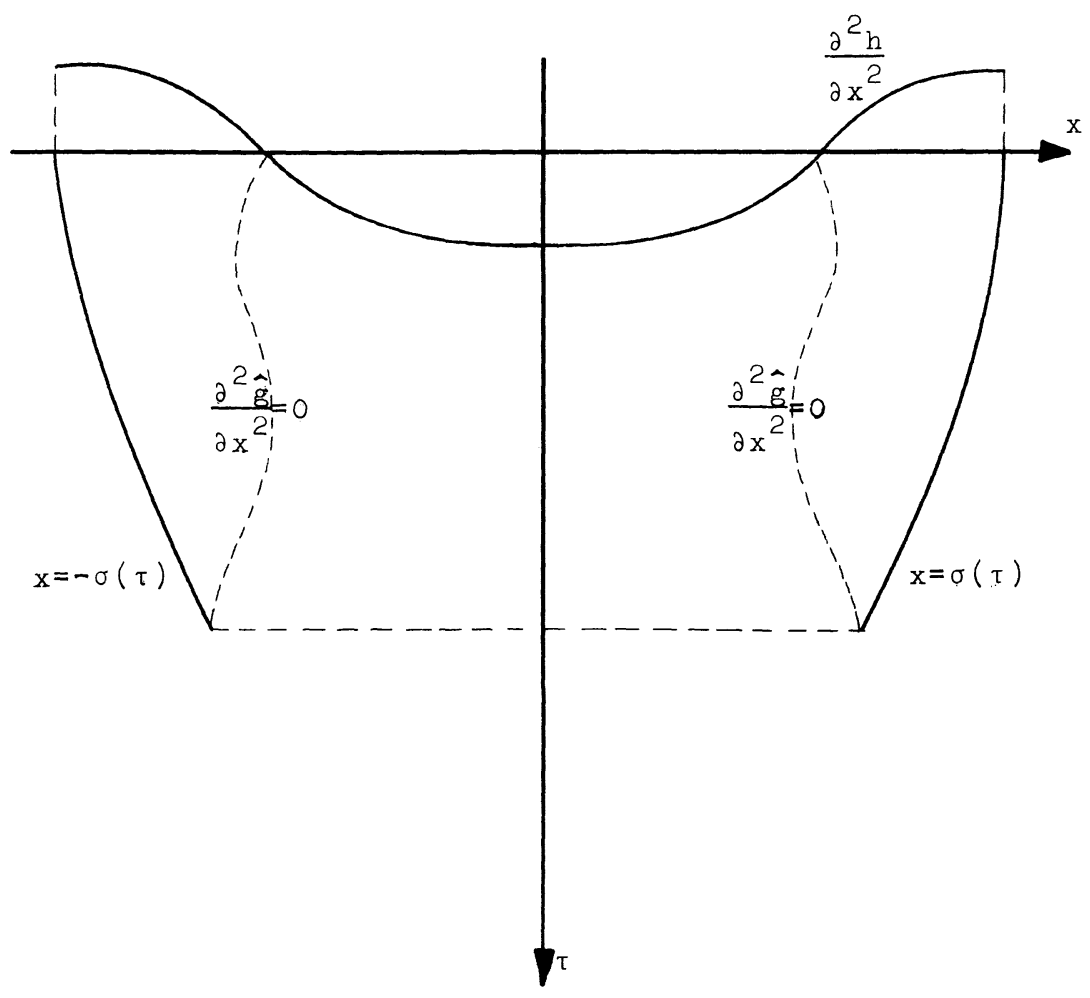

Fig. 17

This argument suggests once more that if the ice-melting picture is to come from an optimal stopping problem, you must actually have the conservation law (26).

It is interesting to discuss the analogy between Stefan's problem and 
optimization with regard to the appearance of holes in the continuation region exposed in $\$ 3.6$. Let

$$
\begin{aligned}
g & =T-t, \quad t<T, \\
& =h(x), \quad t=T,
\end{aligned}
$$

where $h(x)$ has strong enough peaks to generate a hole in the continuation region. The behavior of the water-ice interface is straightforward before the instant $\tau_{1}$ the hole appears (cf. Figure 13). At this moment, someone puts an ice-germ at $x=0$, after which all proceeds freely: this germ of ice grows, shrinks and finally disappears. Again at time $\tau_{2}$ man's intervention is needed, after which things evolve again according to nature.

The Shape of the Boundary Near the Critical Point. Here we assume $g$ as in (22) and in Theorem II; moreover, let $h(x)$ be even. Then it follows from the integral equations for $s(t)$ (similar to (9)) that

$$
\lim _{t \downarrow t_{\infty}} \dot{s}(t)=0 ;
$$

so, in this case, the boundary exhibits a cusp at $t=t_{\infty}$.

\section{REFERENCES}

1. P. Appell, Sur l'équation $\partial^{2} z / \partial x^{2}-\partial z / \partial y=0$ et la théorie de la chaleur, J. Math. Pures Appl. 8 (1892), 187-216.

2. J. A. Bather, Bayes procedure for deciding the sign of a normal mean, Proc. Camb. Philos. Soc. 58 (1962), 599-620.

3. - Optimal stopping problems for brownian motion, Adv. Appl. Prob. 2 (1970), 259-286.

4. A Beurling, An extension of the Riemann mapping theorem, Acta Math. 90 (1953), 117-130.

5. - Conformal mapping and Schlicht functions, seminar, The Institute for Advanced Study, Princeton, N. J.

6. W. Boyce, Stopping rules for selling bonds, The Bell Journal of Economics and Management Science 1 (1970), 27-53.

7. J. V. Breakwell and $\mathrm{H}$. Chernoff, Sequential test for the mean of a normal distribution II (large t), Ann. Math. Statist. 35 (1964), 162-173.

8. L. Breiman, Stopping rule problems, Applied Combinatorial Mathematics (ed. E. F. Bechenbach), Wiley, New York (1964), 284- 319.

9. J. R. Cannon and C. D. Hill, Existence, uniqueness, stability and monotone dependence in a Stefan problem, J. Math. Mech., 17 (1967), 1-14.

10. I. D. Cherkasov, On the transformation of the diffusion process to a Wiener process, Theory of Prob. and its Appl. 2 (1957), 373-377.

11. H. Chernoff, Sequential tests for the mean of a normal distribution, Proc. Fourth Berkeley Symposium 1 (1961), 79-91.

12. - Sequential tests for the mean of a normal distribution III (small t), Ann. Math. Statist. 36 (1965), 28-54. 
13. - Sequential tests for the mean of a normal distribution IV (discrete case), Ann. Math. Statist 36 (1965), 55-68.

14. — Optimal Stochastic control, Sankhya 30 (1968), 221-252.

15. Y. S. Chow, S. Moriguti, H. Robbins, and S. M. Samuels, Optimal selection based on relative rank (the "Secretary" problem), Israel Journal of Mathematics 2, 2 (1964), 81-90.

16. Y. S. Chow and H. Robbins, On optimal stopping rules for $s_{n} / n$, Illinois J. Math. 9 (1965), 444-454.

17. J. L. Doob, A probability approach to the heat equation, Trans. Amer. Math. Soc. 80 (1955), 216-280.

18. - Conditional Brownian motion and the boundary limits of harmonic functions, Bull. Soc. Math. France 85 (1957), 431-458.

19. A. Dvoretzky, Existence and properties of certain optimal stopping rules, Proc. Fifth Berkeley Symp. on Math. Stat. and Prob. 1 (1965).

20. E. B. Dynkin, Optimal selection of stopping time for a Markov process, Dokl. Akad. Nauk USSR, 1502 (1963), 238-240 (Russian); Soviet Math. (English translation) 4 (1963), 627-629.

21. E. B. Dynkin and A. A. Yushkevich, Markov processes, theorems and problems, Plenum Press, New York, 1969.

22. A. Friedman, Free boundary problems for parabolic equations I: . Melting of Solids, J. Math. and Mech. 8 (1959), 499-518.

23. H. Fölmer, Optimal stopping of constrained brownian motion, J. Appl. Prob. 9 (1972), 557-571.

24. M. Gardner, Mathematical games, Scientific American, 2021 (1960), 150-156; 202, 3 (1960), 173-182.

25. D. S. Griffeath and J. L. Snell, Application of discrete potential theory: the casino and the stock market problems, 1971, to appear.

26. B. I. Grigelionis and A. N. Shiryaev, On Stefan's problem and optimal stopping rules for Markov processes, Theory of Probability and its Applicaons 9 (1966), 541-558.

27. K. Ito and H. P. McKean, Diffusion Processes and Their Sample Paths, Springer Verlag, New York, 1965.

28. M. Kac, Can one hear the shape of a drum, Amer. Math. Monthly 73 (1966), 1-23.

29. I. I. Kolodner, Free boundary problcms for the heat equation with applications to problems of change of phase, Comm. Pure Appl. Math. 9 (1956), $1-31$.

30. D. V. Lindley, Dynamic programming and decision theory, Applied Statistics 10 (1961), 81-90.

31. W. L. Miranker, A free boundary value problem for the heat equation, Quarterly of Applied Mathematics, 16, 2 (1958), 121-130.

32. H. P. McKean, Stochastic Integrals, Academic Press, 1971.

33. —, Appendix: A free boundary problem for the heat equation arising from a problem in mathematical economics, Industrial Management Review, 6, 2 (1965), 32-39.

34. S. Moriguti and H. Robbins, A Bayes test of " $p \leqslant 1 / 2$ " versus " $p>1 / 2$ ", Rep. Stat. Appl. Res., JUSE, 9, 2 (1962), 39-60.

35. H. Robbins, Optimal stopping, Amer. Math. Monthly, April (1970), 333-343.

36. H. Robbins and D. O. Siegmund, Boundary crossing probabilities for the Wiener Process, Tech report No. 4, June 23, 1969. 
37. —, Great Expectations: The Theory of Optimal Stopping, Houghton Mifflin, Boston, 1970.

38. L. I. Rubinstein, Problema Stefana (Russian), Riga, 1967. (Translation to appear in Translations of Mathematical Monographs, Amer. Math. Soc.).

39. P. A. Samuelson, Rational theory of warrant pricing, Industrial Management Review, 6, 2 (1965), 13-31.

40. A. Schatz, Free boundary problems of Stefan type with prescribed flux, J. of Math. Anal. and Appl. 28, 3 (1969), 569-580.

41. L. A. Shepp, Explicit solutions to some problems of optimal stopping, The Annals of Math. Statist., 40, 3 (1969), 993-1010.

42. A. N. Sirjaev, Statistical Sequential Analysis, Moscow, 1969, in Russian, translation to appear.

43. J. L. Snell, Applications of martingale system theorems, Trans. Amer. Math. Soc. 73 (1952), 293-312.

44. H. M. Taylor, Optimal stopping in a Markov process, Ann. Math. Statist. 39 (1968), 1333-1344.

45. H. Teicher and J. Wolfowitz, Existence of stopping rule for linear and quadratic rewards, Z. für Wahrsch. Verw. Geb. 5 (1966), 361-368.

46. P. L. J. van Moerbeke, About optimal stopping and free boundary problems, Archive for Rat. Mech. and Anal., 1974 (to appear).

47. _ An optimal stopping problem with linear reward, Acta Mathematica, 1-2, 132 (1974), 111-151.

48. L. H. Walker, Regarding stopping rules for brownian motion and random walks, Bull. Amer. Math. Soc. 75 (1969), 46-50.

UNIVERSITY OF LouvaIN, LoUVAIN-LA-NeUVE, BELgIUM

Stanford University, Stanford, California 94305 
Table 1

\begin{tabular}{c|cccccccccccccc}
$N^{S_{0}}$ & 9 & 8 & 7 & 6 & 5 & 4 & 3 & 2 & 1 & 0 & -1 & -2 & -3 & -4 \\
\hline 1 & 1 & 1 & 1 & 1 & 1 & 1 & 1 & 1 & 1 & 0 & 0 & 0 & 0 & 0 \\
2 & 2 & 2 & 2 & 2 & 2 & 2 & 2 & 2 & 1.5 & 0.5 & 0 & 0 & 0 & 0 \\
3 & 3 & 3 & 3 & 3 & 3 & 3 & 3 & 2.75 & 2.25 & 0.75 & 0.25 & 0 & 0 & 0 \\
4 & 4 & 4 & 4 & 4 & 4 & 4 & 3.875 & 3.625 & 2.75 & 1.25 & 0.375 & 0 & 0 & 0 \\
5 & 5 & 5 & 5 & 5 & 5 & 4.937 & 4.812 & 4.312 & 3.437 & 1.562 & 0.625 & 0 & 0 & 0 \\
6 & 6 & 6 & 6 & 6 & 5.968 & 5.906 & 5.625 & 5.125 & 3.937 & 2.031 & 0.781 & 0 & 0 & 0 \\
7 & 7 & 7 & 7 & 6.984 & 6.984 & 6.796 & 6.515 & 5.781 & 4.578 & 2.354 & 1.015 & 0 & 0 & 0 \\
8 & 8 & 8 & 7.992 & 7.976 & 7.890 & 7.734 & 7.289 & 6.546 & 5.070 & 2.796 & 1.179 & 0 & 0 & 0 \\
9 & 9 & 8.996 & 8.988 & 8.941 & 8.855 & 8.589 & 8.140 & 7.179 & 5.671 & 3.125 & 1.398 & 0 & 0 & 0 \\
10 & & 9.994 & 9.968 & 9.921 & 9.765 & 9.498 & 8.884 & 7.906 & 6.152 & 3.535 & 1.562 & 0 & 0 & 0 \\
11 & & & 10.958 & 10.867 & 10.709 & 10.325 & 9.702 & 8.518 & 6.720 & 3.857 & 1.767 & 0 & 0 & 0 \\
12 & & & & 11.833 & 11.596 & 11.206 & 10.421 & 9.211 & 7.187 & 4.244 & 1.928 & 0 & 0 & 0 \\
13 & & & & & 12.520 & 12.009 & 11.208 & 9.804 & 7.727 & 4.558 & 2.122 & 0 & 0 & 0 \\
14 & & & & & & 12.864 & 11.906 & 10.468 & 8.181 & 4.924 & 2.279 & 0.061 & 0 & 0 \\
15 & & & & & & & 12.666 & 11.044 & 8.696 & 5.230 & 2.462 & 0.139 & 0 & 0 \\
16 & & & & & & & & 11.681 & 9.137 & 5.579 & 2.684 & 0.231 & 0 & 0 \\
17 & & & & & & & & & 9.630 & 6.911 & 2.905 & 0.342 & 0 & 0 \\
18 & & & & & & & & & & 7.268 & 3.626 & 0.623 & 0 & 0 \\
19 & & & & & & & & & & & 3.946 & 0.813 & 0 & 0 \\
20 & & & & & & & & & & & & 0.973 & 0 & 0 \\
\hline
\end{tabular}

